\title{
Observation of an Inversion in Photophysical Tuning in a Systematic Study of Luminescent Triazole-Based Osmium(II) Complexes
}

\author{
Paul A. Scattergood,* James Roberts, Salem A. E. Omar and Paul I. P. Elliott* \\ Department of Chemistry, University of Huddersfield, Queensgate, Huddersfield, HD1 3DH, UK
}

\section{Figure S1}

${ }^{1} \mathrm{H}$ NMR spectrum of ligand $\mathbf{3}$

Figure S2

${ }^{13} \mathrm{C}$ NMR spectrum of ligand $\mathbf{3}$

S4

Figure S3

High resolution ESI mass spectrum of ligand $\mathbf{3}$

S5

Figure S4

${ }^{1} \mathrm{H}$ NMR spectrum of ligand 7

S6

Figure S5

${ }^{13} \mathrm{C}$ NMR spectrum of ligand 7

S7

Figure S6

High resolution ESI mass spectrum of ligand 7

${ }^{1} \mathrm{H}$ NMR spectrum of ligand $\mathbf{1 0}$

Figure S7

Figure S8

${ }^{13} \mathrm{C}$ NMR spectrum of ligand $\mathbf{1 0}$

S8

S9

Figure S9

High resolution ESI mass spectrum of ligand $\mathbf{1 0}$

S10

Figure S10

${ }^{1} \mathrm{H}$ NMR spectrum of ligand $\mathbf{1 4}$

S11

Figure S11

${ }^{13} \mathrm{C}$ NMR spectrum of ligand $\mathbf{1 4}$

S12

Figure S12

High resolution ESI mass spectrum of ligand $\mathbf{1 4}$

S13

Figure S13

${ }^{1} \mathrm{H}$ NMR spectrum of ligand $\mathbf{1 7}$

${ }^{13} \mathrm{C}$ NMR spectrum of ligand $\mathbf{1 7}$

S14

S15

Figure S14

High resolution ESI mass spectrum of ligand $\mathbf{1 7}$

S16

Figure S15

${ }^{1} \mathrm{H}$ NMR spectrum of complex Os2

S17

Figure S16

${ }^{13} \mathrm{C}$ NMR spectrum of complex Os2

S18

Figure S17

Figure S18

High resolution ESI mass spectrum of complex Os2

S19

Figure S19

${ }^{1} \mathrm{H}$ NMR spectrum of complex $\mathbf{O s 3}$

S20

Figure S20

${ }^{13} \mathrm{C}$ NMR spectrum of complex $\mathbf{O s 3}$

S21

S22

Figure S21

High resolution ESI mass spectrum of complex Os3

S23

Figure S22

${ }^{1} \mathrm{H}$ NMR spectrum of complex Os4

S24

${ }^{13} \mathrm{C}$ NMR spectrum of complex Os4

S25

High resolution ESI mass spectrum of complex Os4

S26

Figure S24

${ }^{1} \mathrm{H}$ NMR spectrum of complex $\mathbf{O s 5}$

S27

Figure S25

${ }^{13} \mathrm{C}$ NMR spectrum of complex $\mathbf{O s 5}$

S28

Figure S27

High resolution ESI mass spectrum of complex Os5

S29

Figure S28

${ }^{1} \mathrm{H}$ NMR spectrum of complex Os6

S30

Figure S29

${ }^{13} \mathrm{C}$ NMR spectrum of complex Os6

S31

Figure S30

High resolution ESI mass spectrum of complex Os6

S32

Figure S31

77 K Photoluminescence spectra for Os1-Os6

S33

Figure S32

Additional electrochemical data for complex Os4

S33

Figure S33 
Optimised ground state geometry atomic coordinates for Os1 


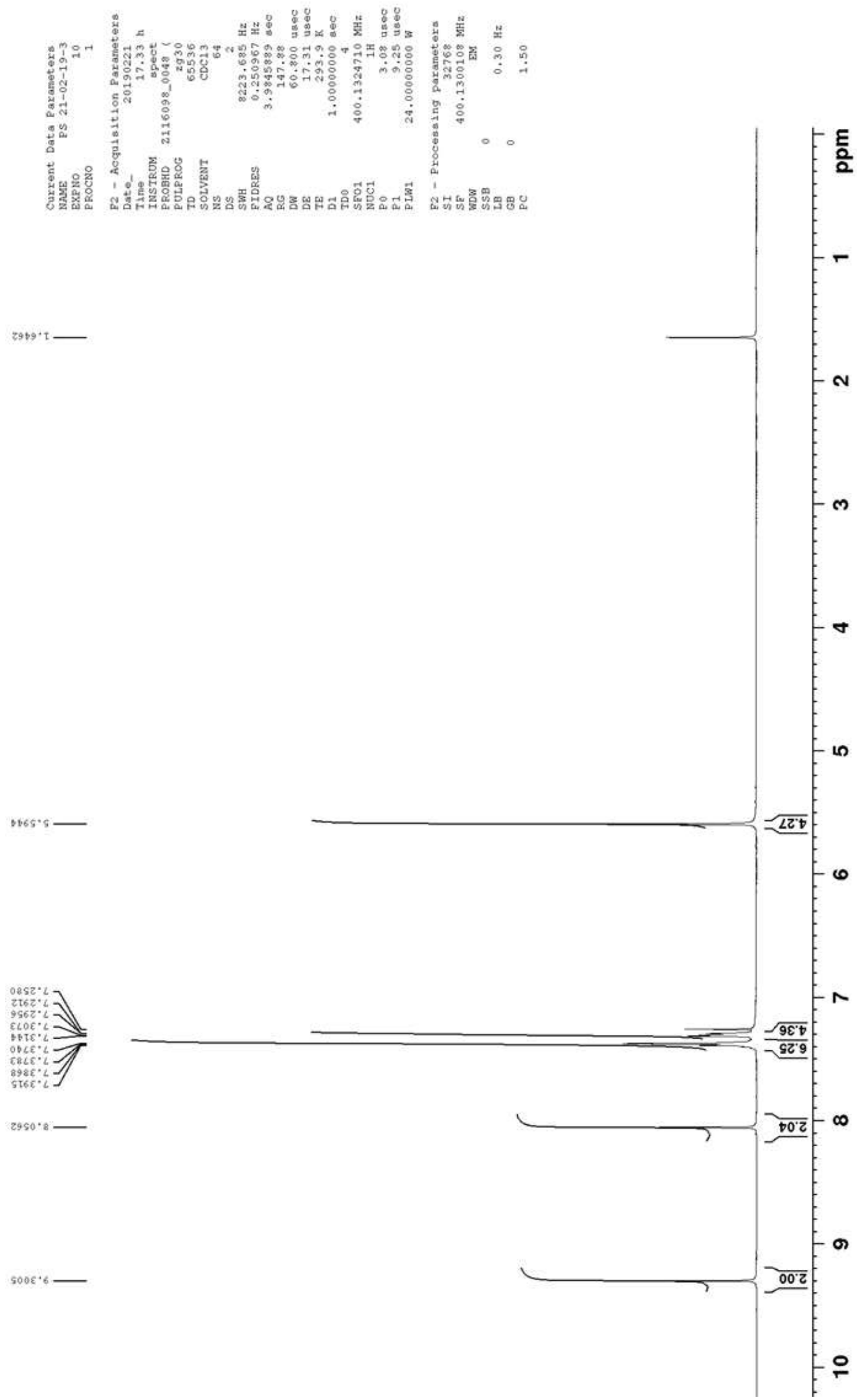

Figure S1 $\quad{ }^{1} \mathrm{H}$ NMR spectrum $\left(\mathrm{CDCl}_{3}, 400 \mathrm{MHz}\right)$ of ligand 3. 


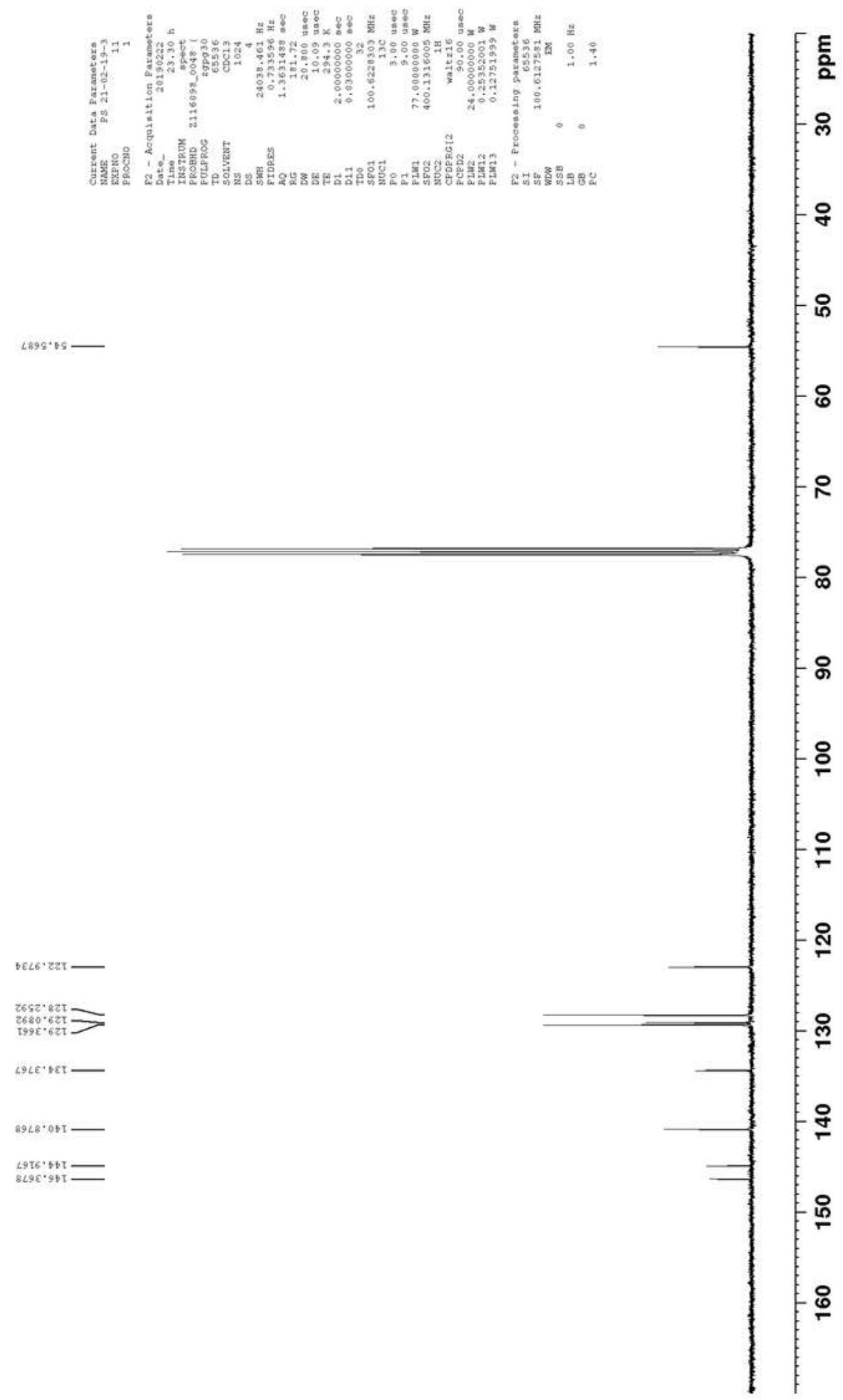

Figure S2 $\quad{ }^{13} \mathrm{C}$ NMR spectrum $\left(\mathrm{CDCl}_{3}, 101 \mathrm{MHz}\right)$ of ligand 3. 


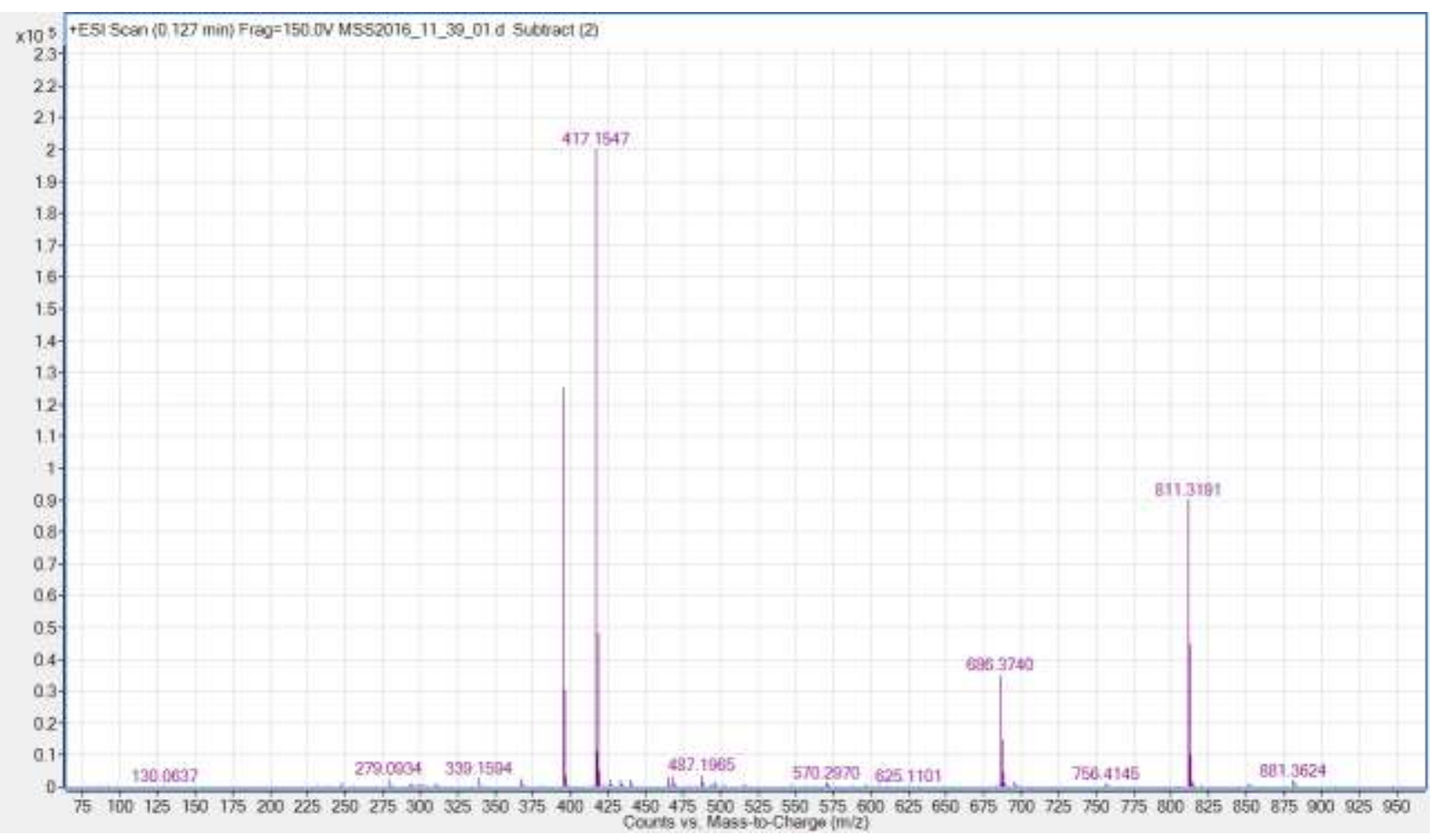

Figure S3 High resolution ESI mass spectrum of ligand 3. 


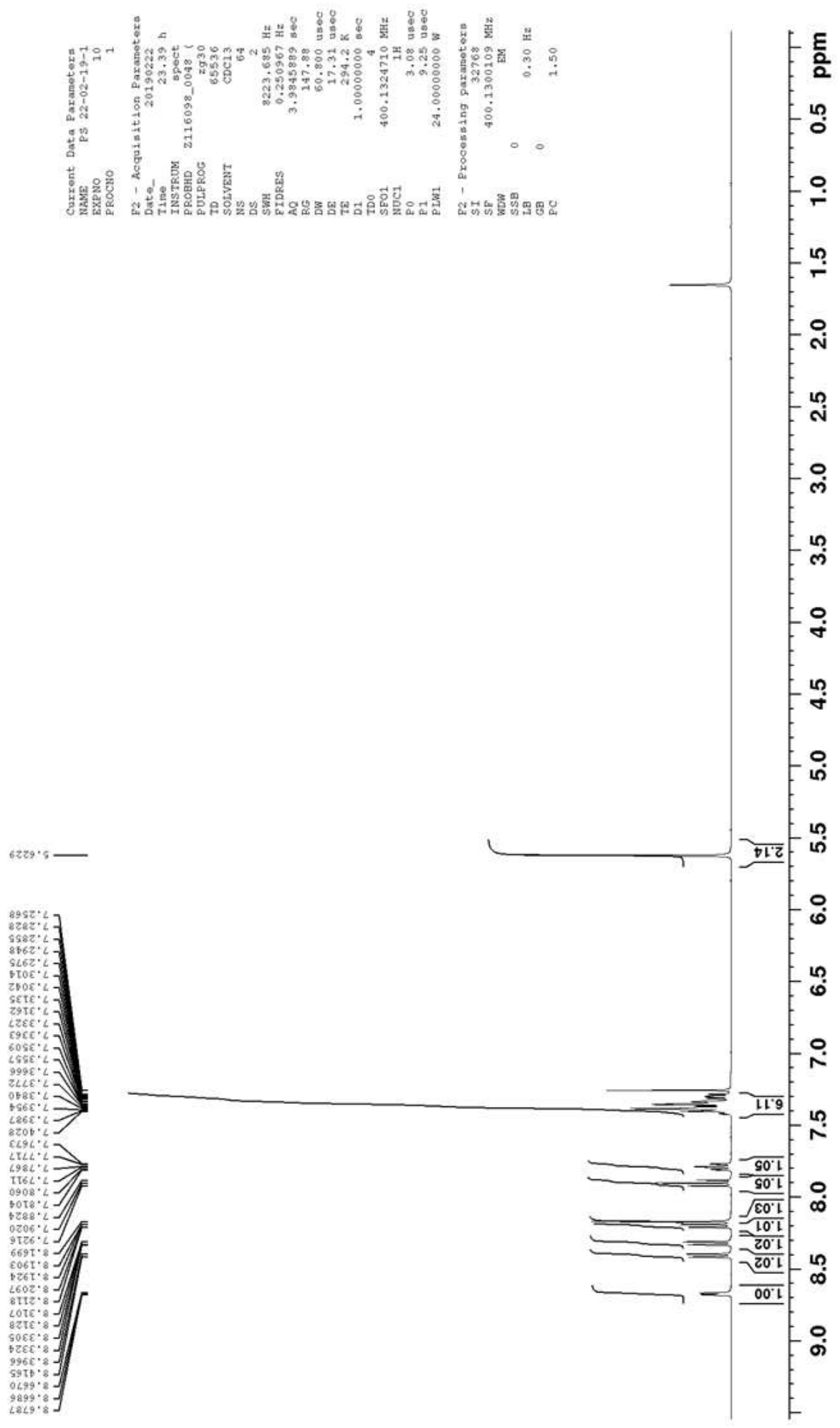

Figure $\mathbf{S 4} \quad{ }^{1} \mathrm{H}$ NMR spectrum $\left(\mathrm{CDCl}_{3}, 400 \mathrm{MHz}\right)$ of ligand 7. 


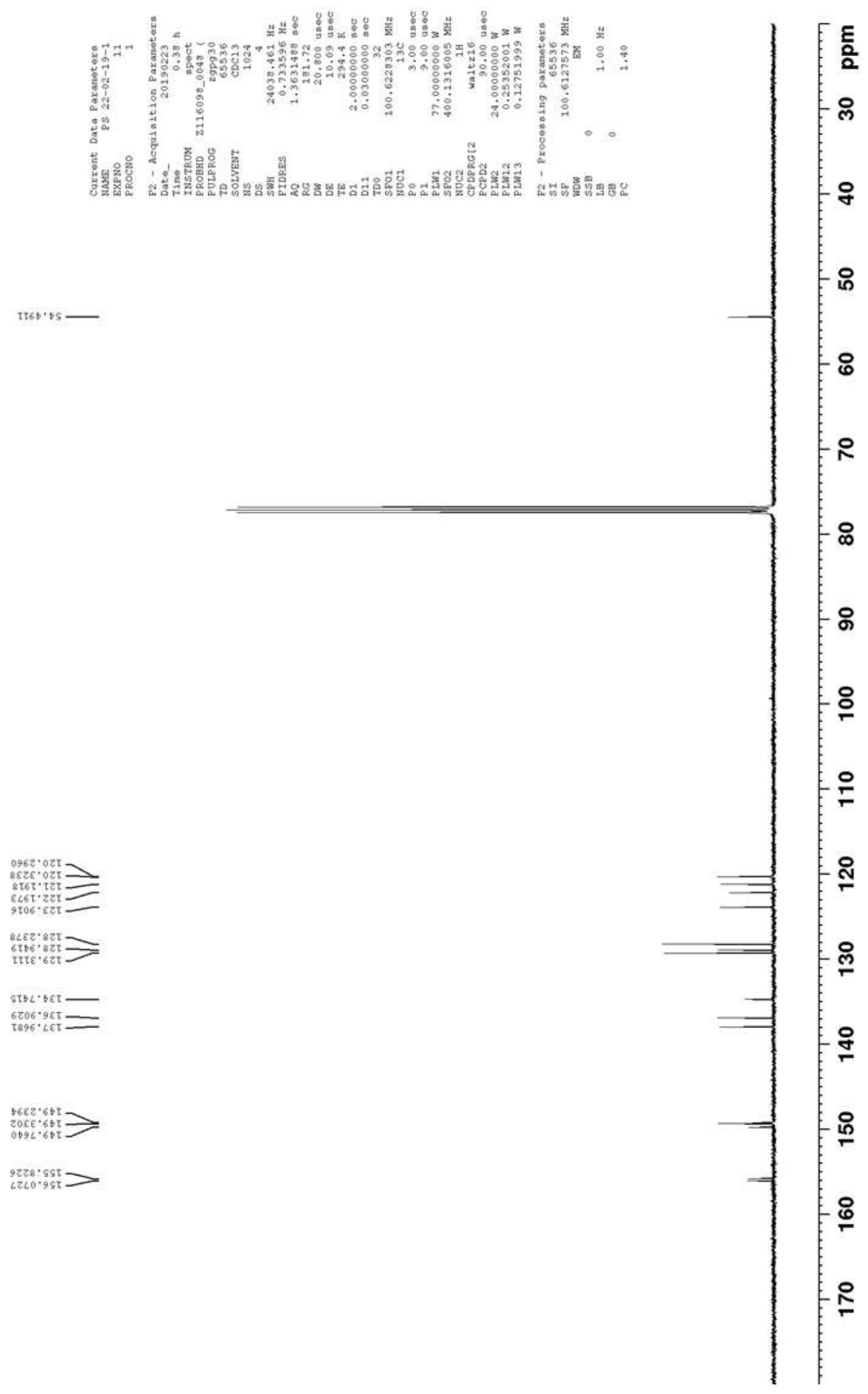

Figure S5 $\quad{ }^{13} \mathrm{C}$ NMR spectrum $\left(\mathrm{CDCl}_{3}, 101 \mathrm{MHz}\right)$ of ligand 7. 


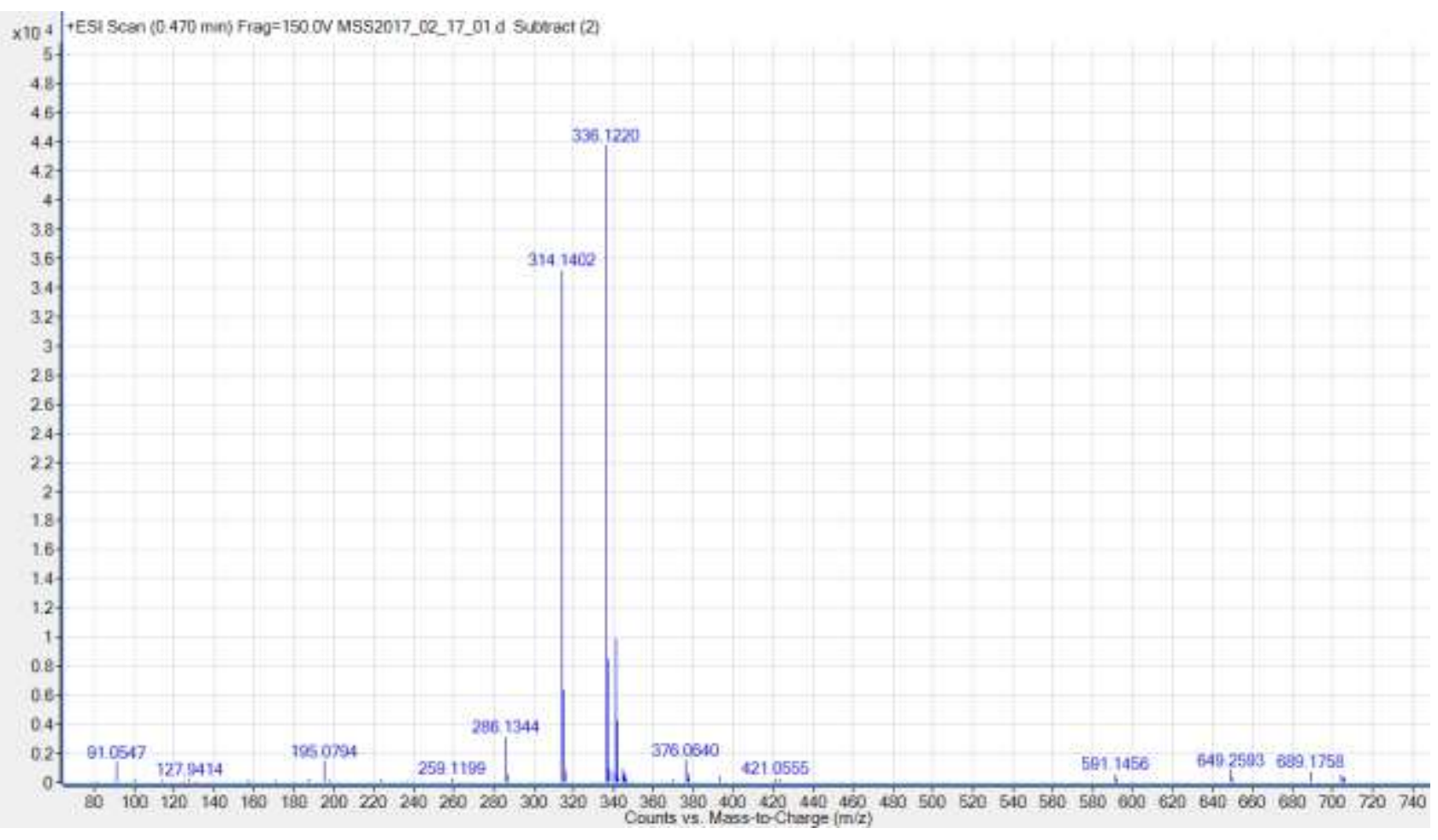

Figure S6 High resolution ESI mass spectrum of ligand 7. 

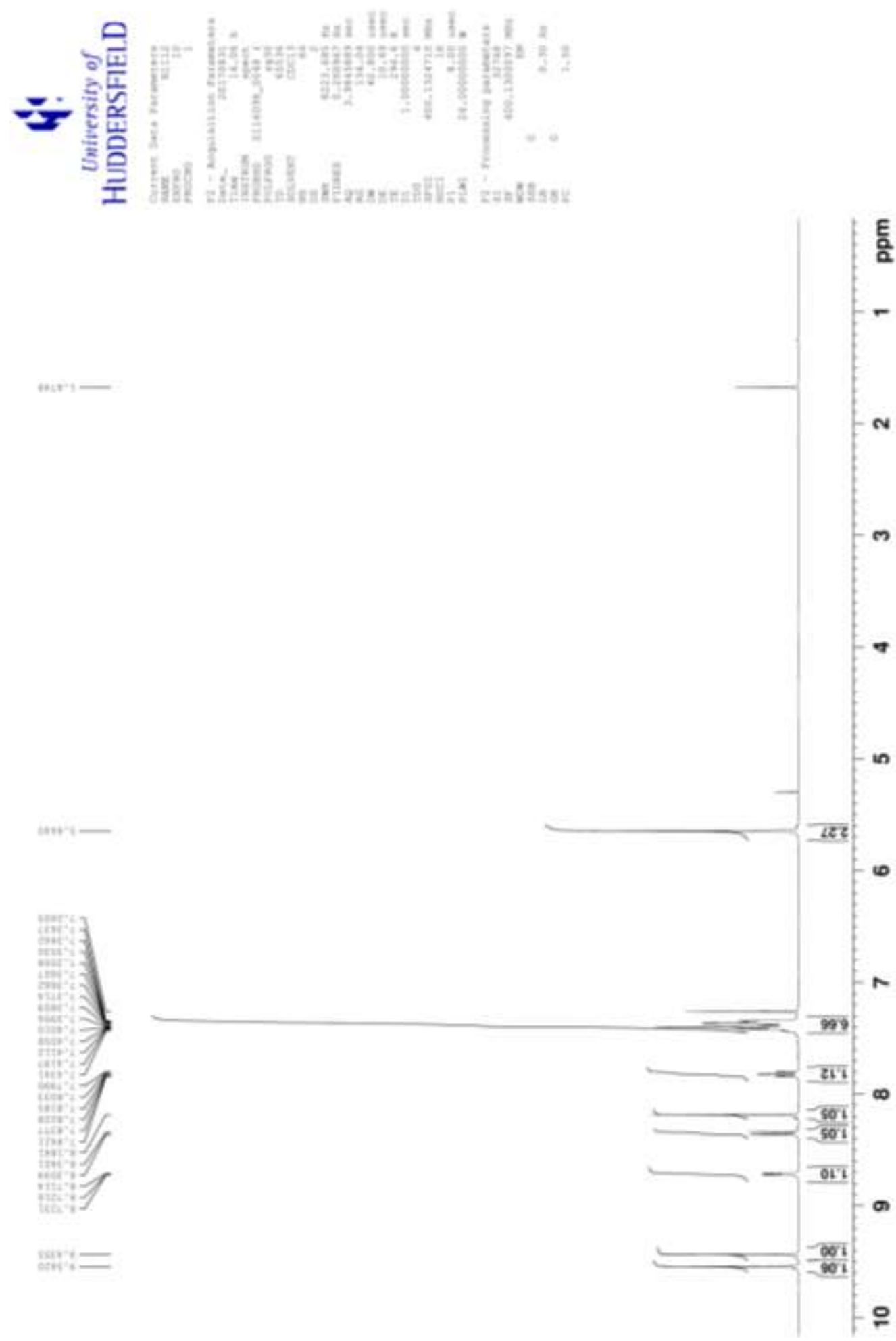

Figure S7 $\quad{ }^{1} \mathrm{H}$ NMR spectrum $\left(\mathrm{CDCl}_{3}, 400 \mathrm{MHz}\right)$ of ligand 10. 

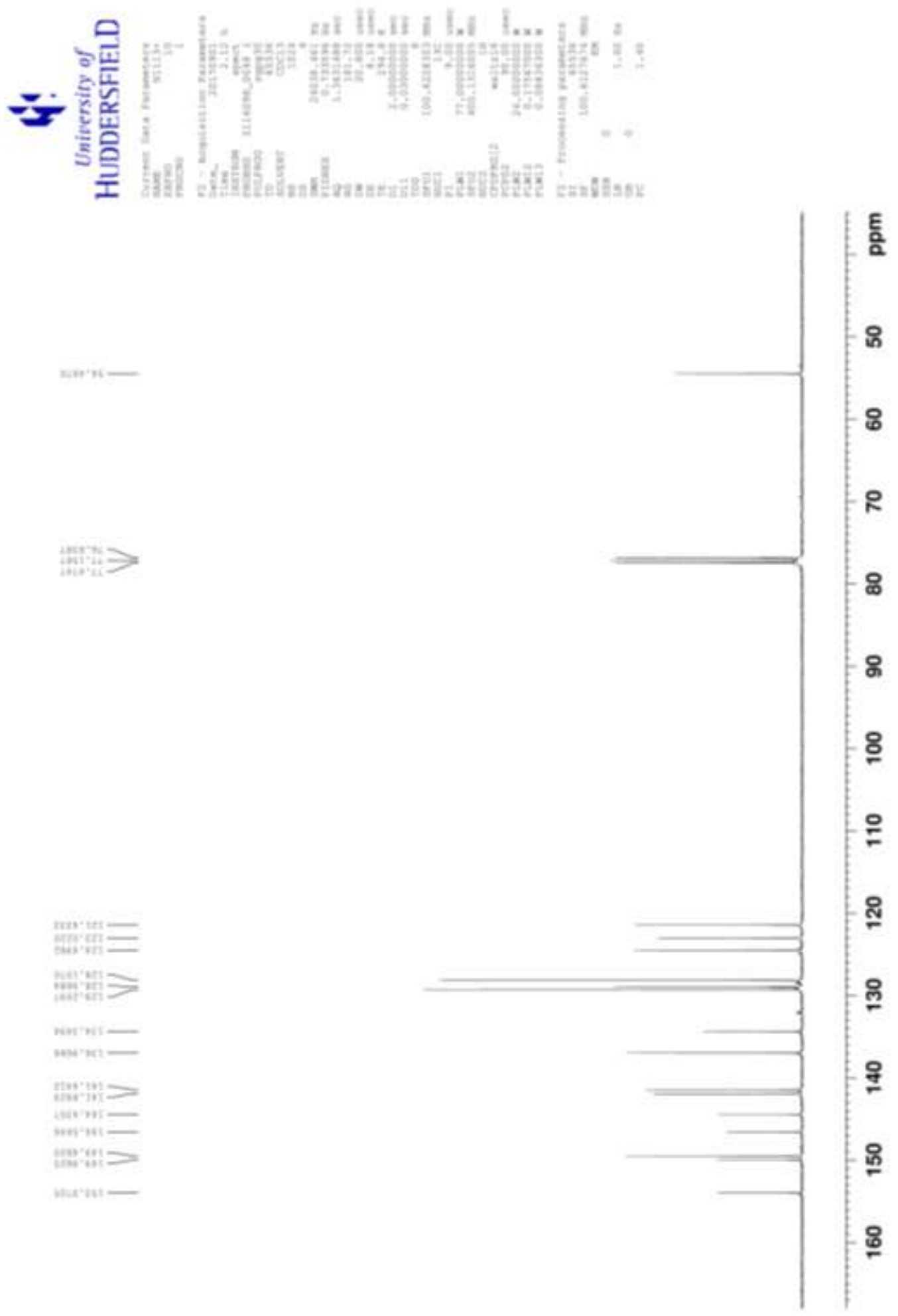

Figure S8 $\quad{ }^{13} \mathrm{C}$ NMR spectrum $\left(\mathrm{CDCl}_{3}, 101 \mathrm{MHz}\right)$ of ligand $\mathbf{1 0}$. 


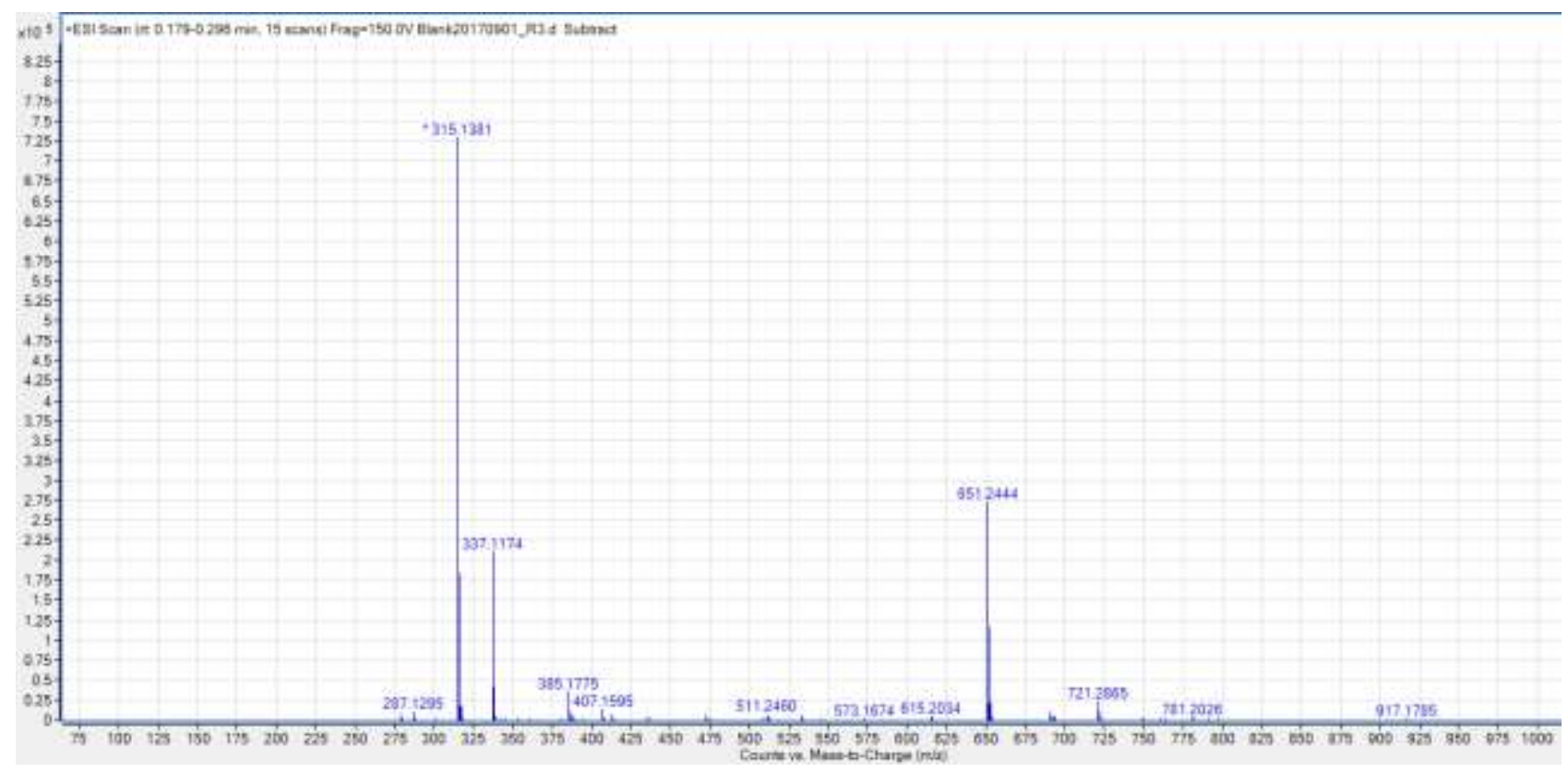

Figure S9 High resolution ESI mass spectrum of ligand $\mathbf{1 0 .}$ 

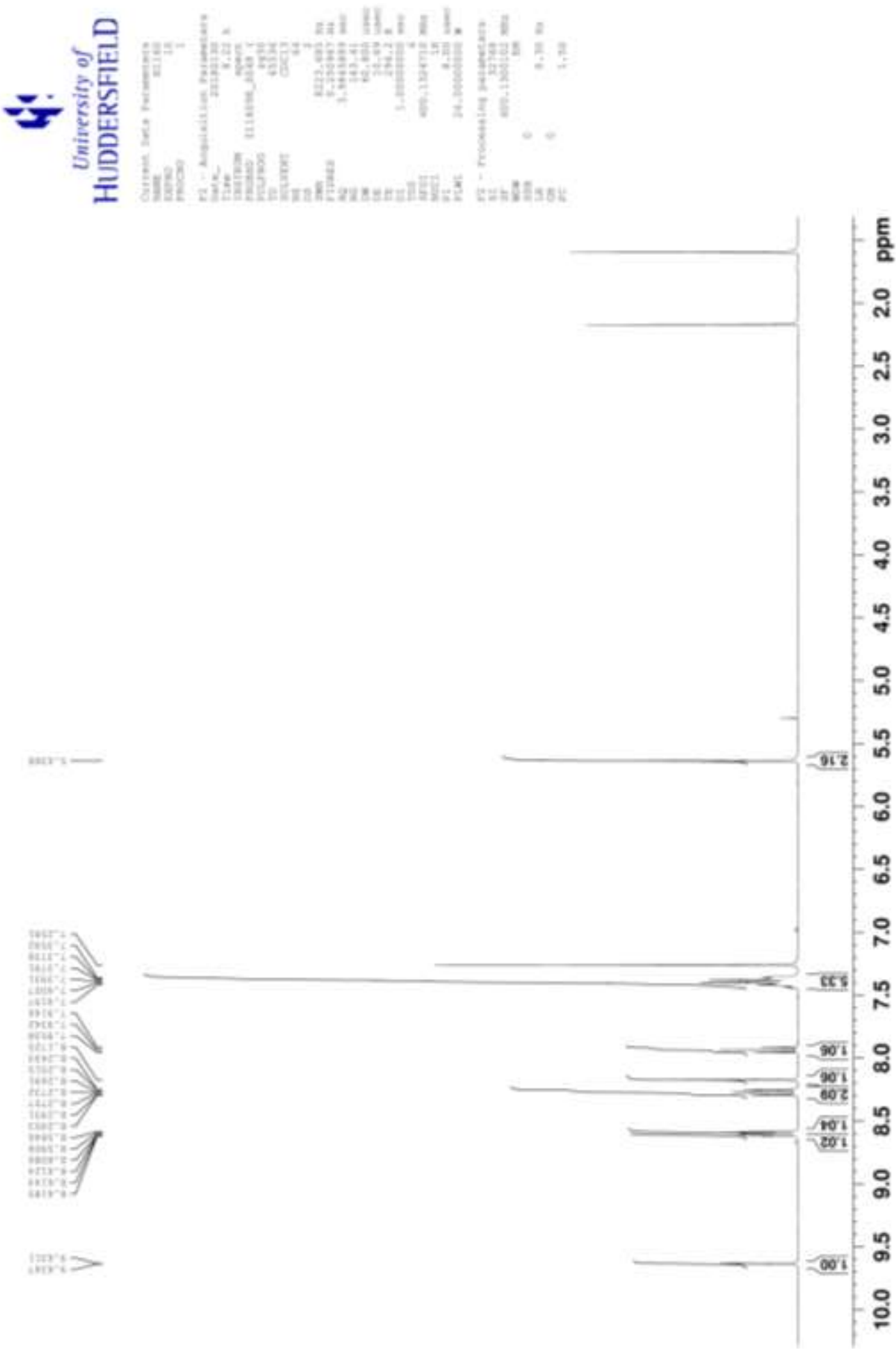

Figure S10 $\quad{ }^{1} \mathrm{H}$ NMR spectrum $\left(\mathrm{CDCl}_{3}, 400 \mathrm{MHz}\right)$ of ligand 14. 


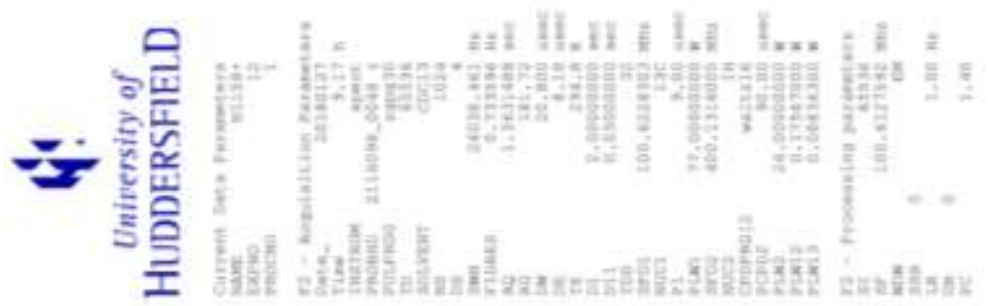

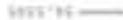

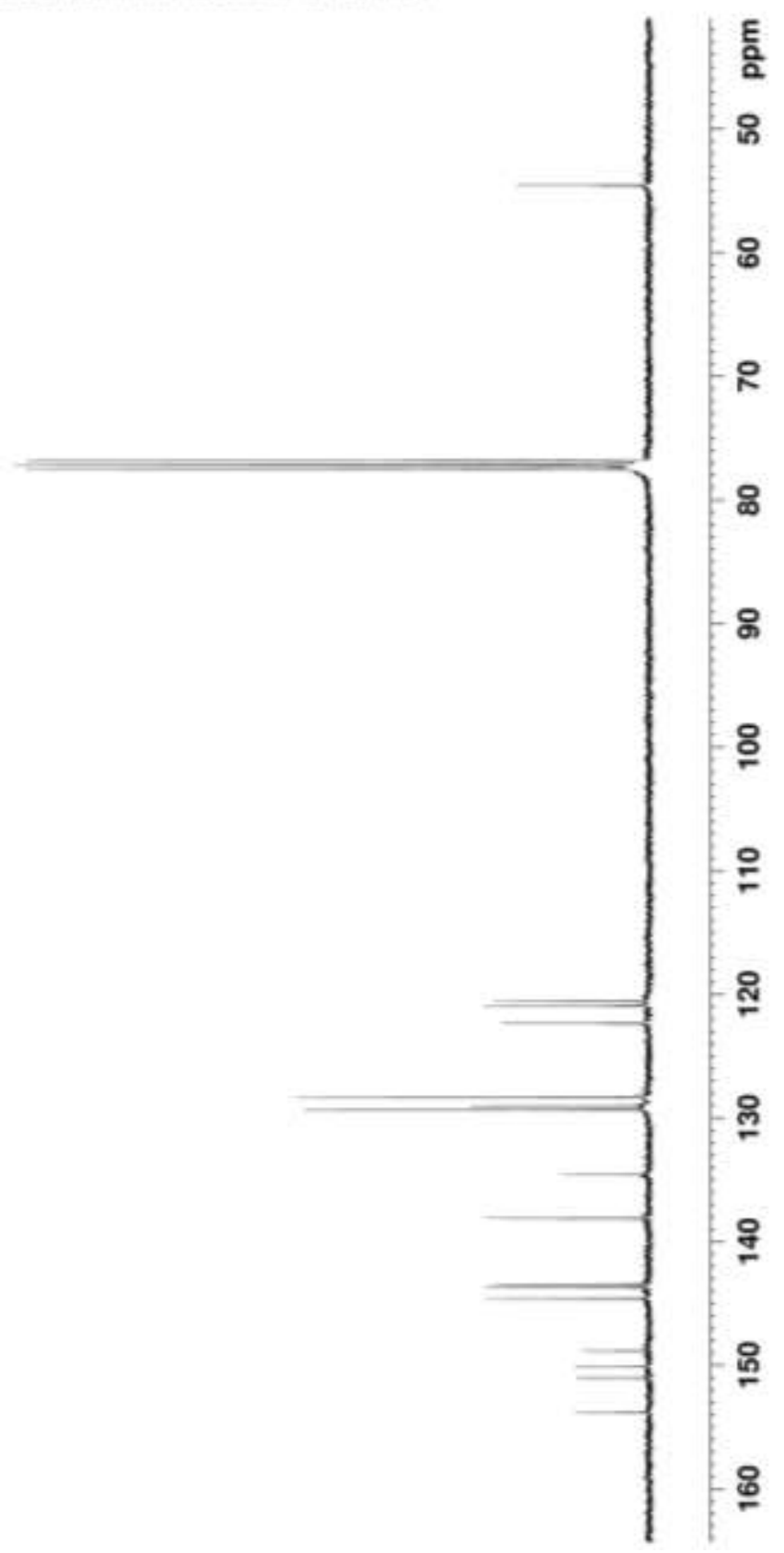

Figure S11 $\quad{ }^{13} \mathrm{C}$ NMR spectrum $\left(\mathrm{CDCl}_{3}, 400 \mathrm{MHz}\right)$ of ligand 14. 


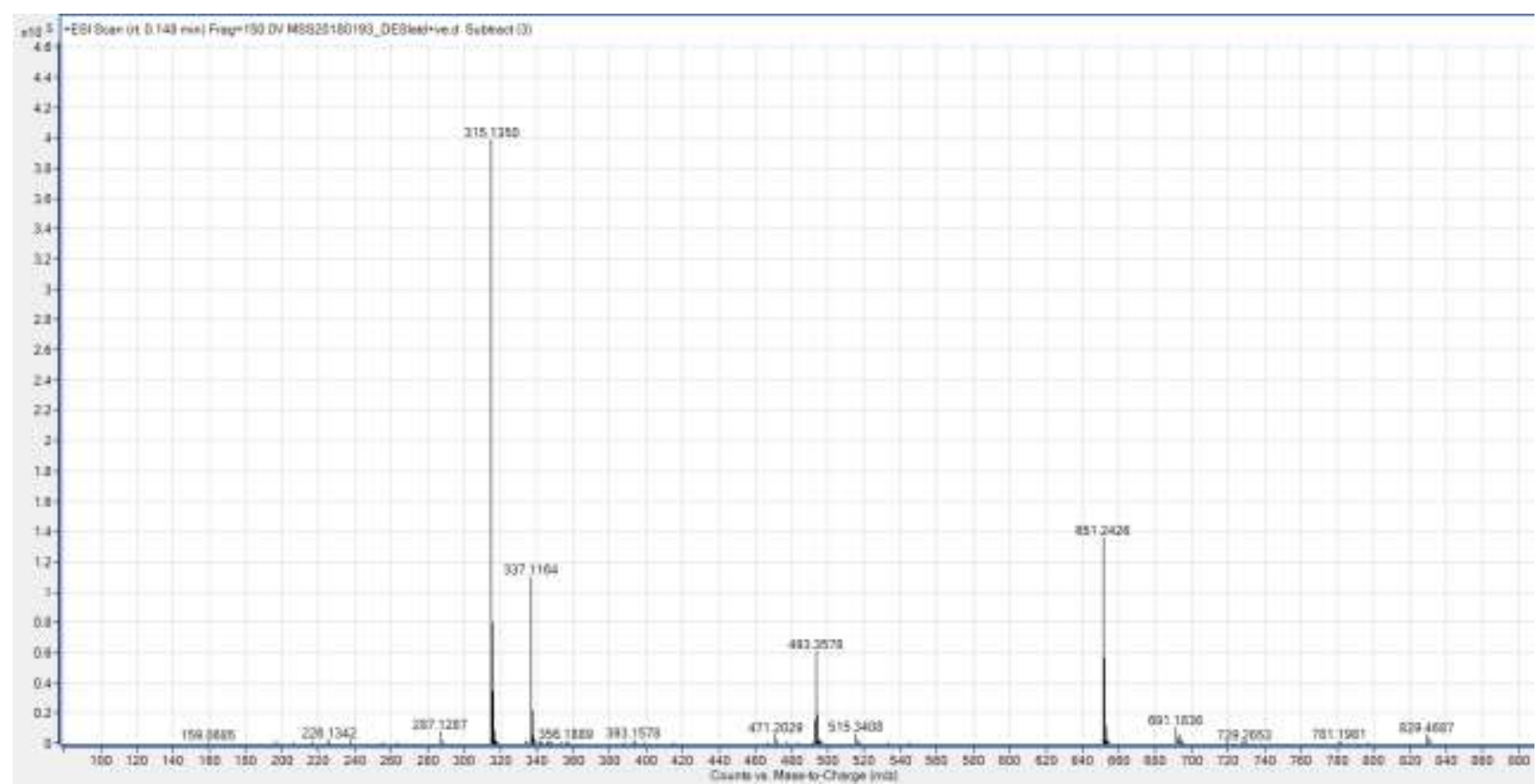

Figure S12 High resolution ESI mass spectrum for ligand 14. 


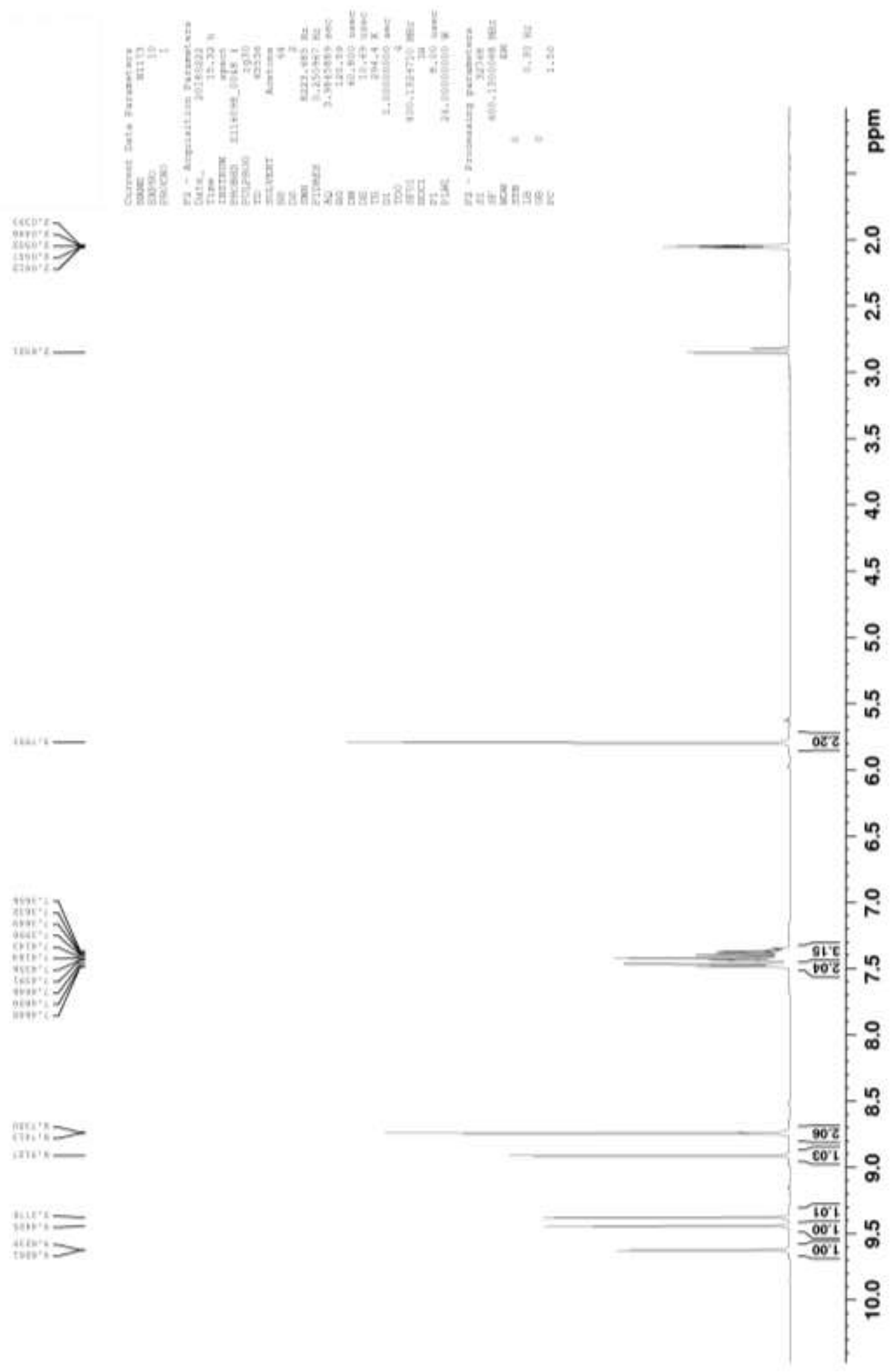

Figure S13 $\quad{ }^{1} \mathrm{H}$ NMR spectrum (d6-Acetone, $\left.400 \mathrm{MHz}\right)$ of ligand 17. 

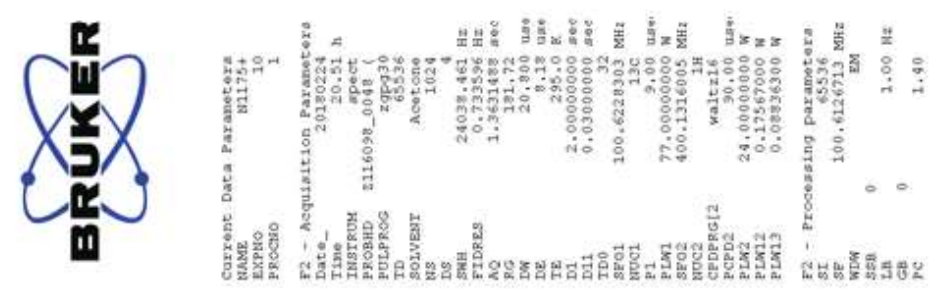

$\tau L \cdot b \varsigma-$
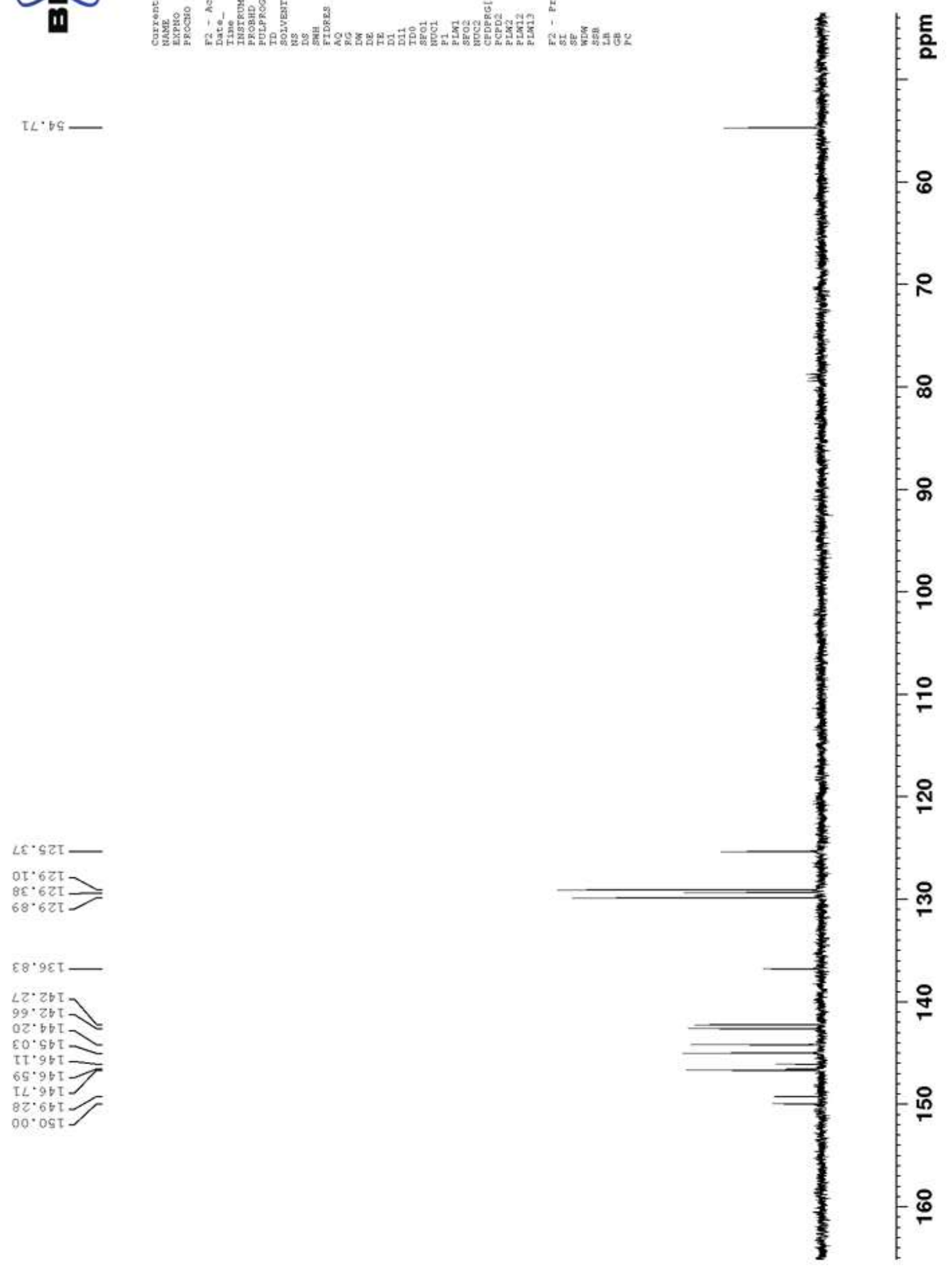

Figure S14 $\quad{ }^{13} \mathrm{C}$ NMR spectrum ( $\mathrm{d}_{6}$-Acetone, $\left.101 \mathrm{MHz}\right)$ of ligand 17. 


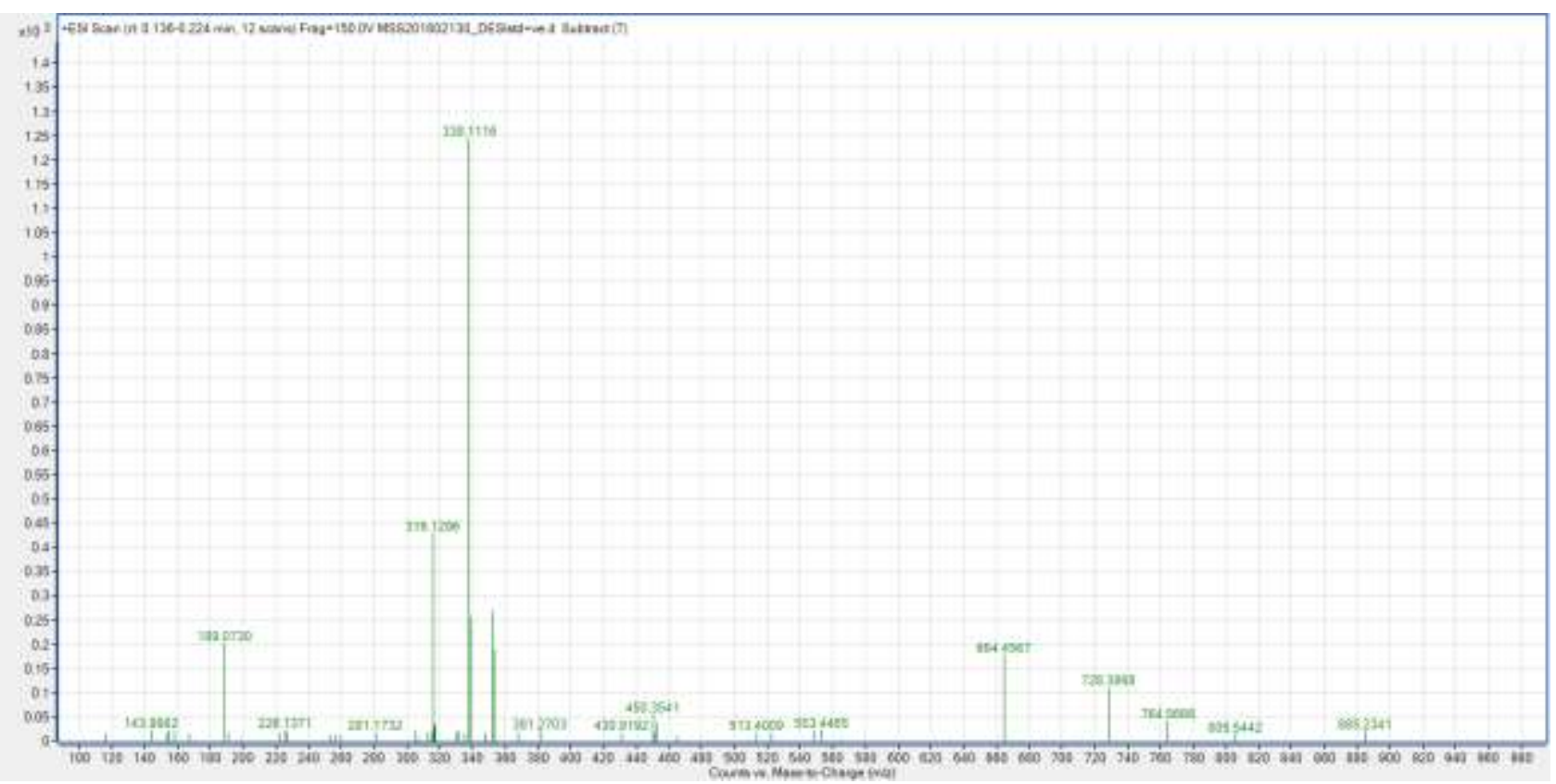

Figure S15 High resolution ESI mass spectrum for ligand 17. 


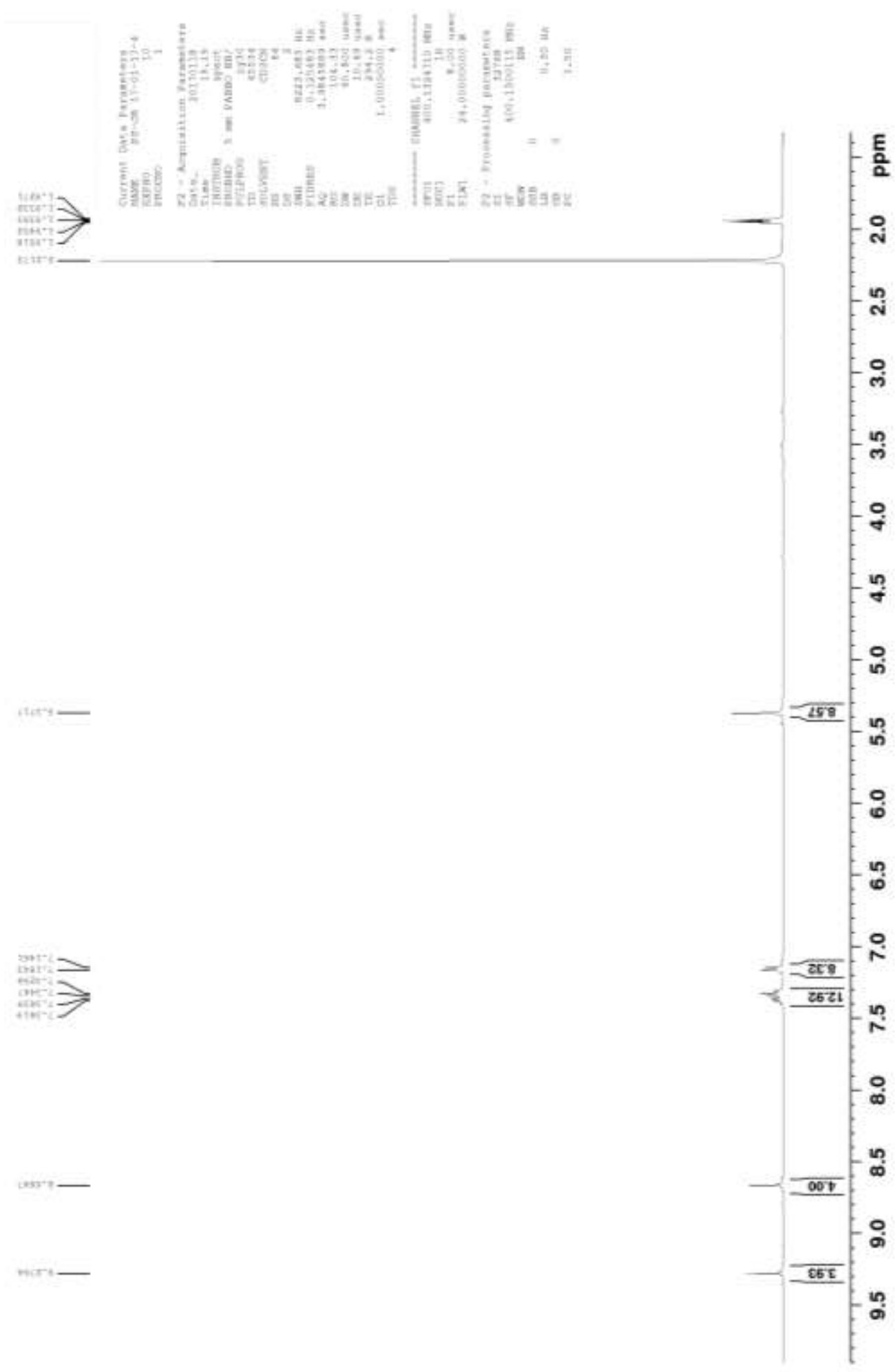

Figure S16 $\quad{ }^{1} \mathrm{H}$ NMR spectrum $\left(\mathrm{d}_{3}-\mathrm{MeCN}, 400 \mathrm{MHz}\right)$ of complex Os2. 


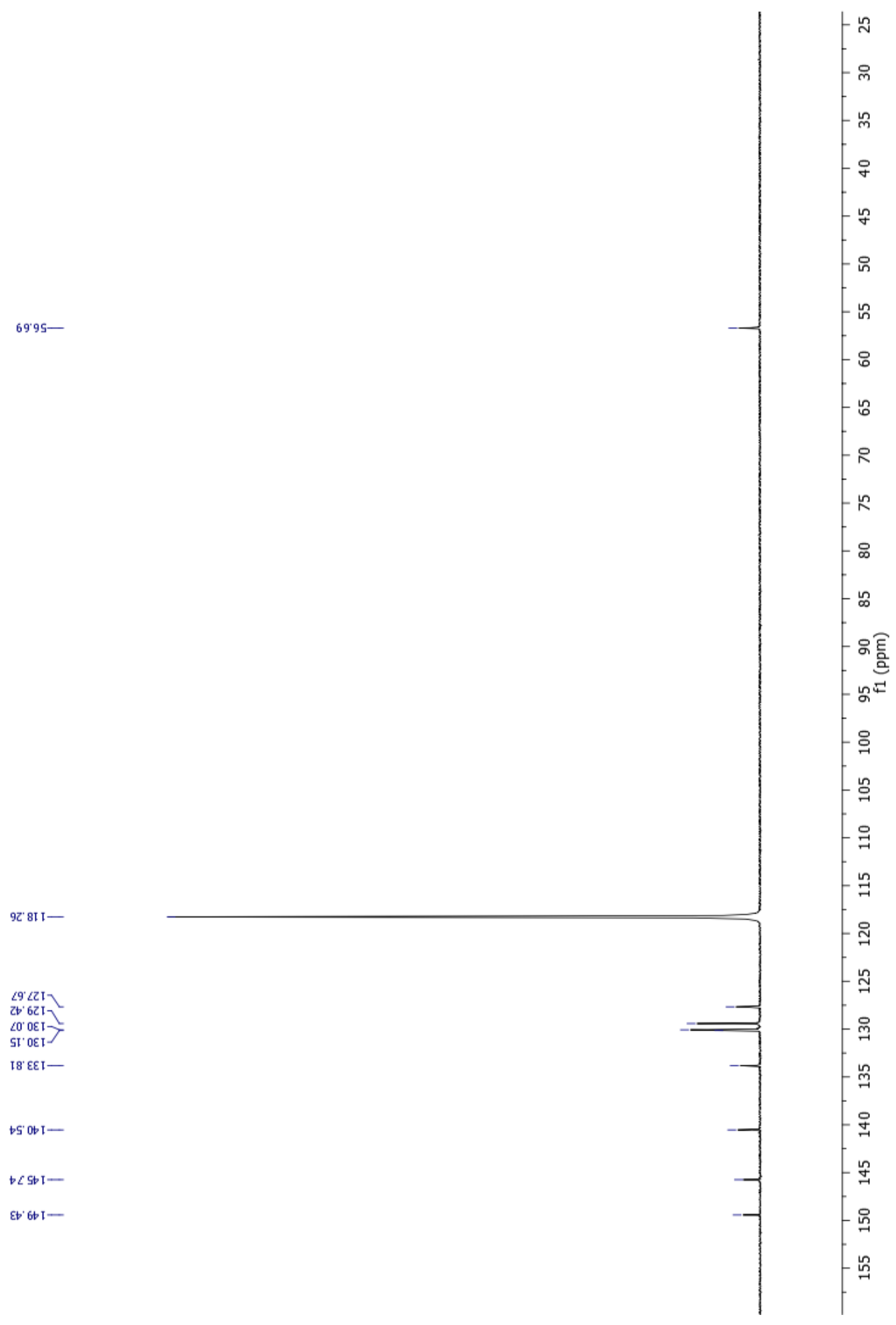

Figure S17 $\quad{ }^{13} \mathrm{C}$ NMR spectrum $\left(\mathrm{d}_{3}-\mathrm{MeCN}, 400 \mathrm{MHz}\right)$ of complex Os2. 


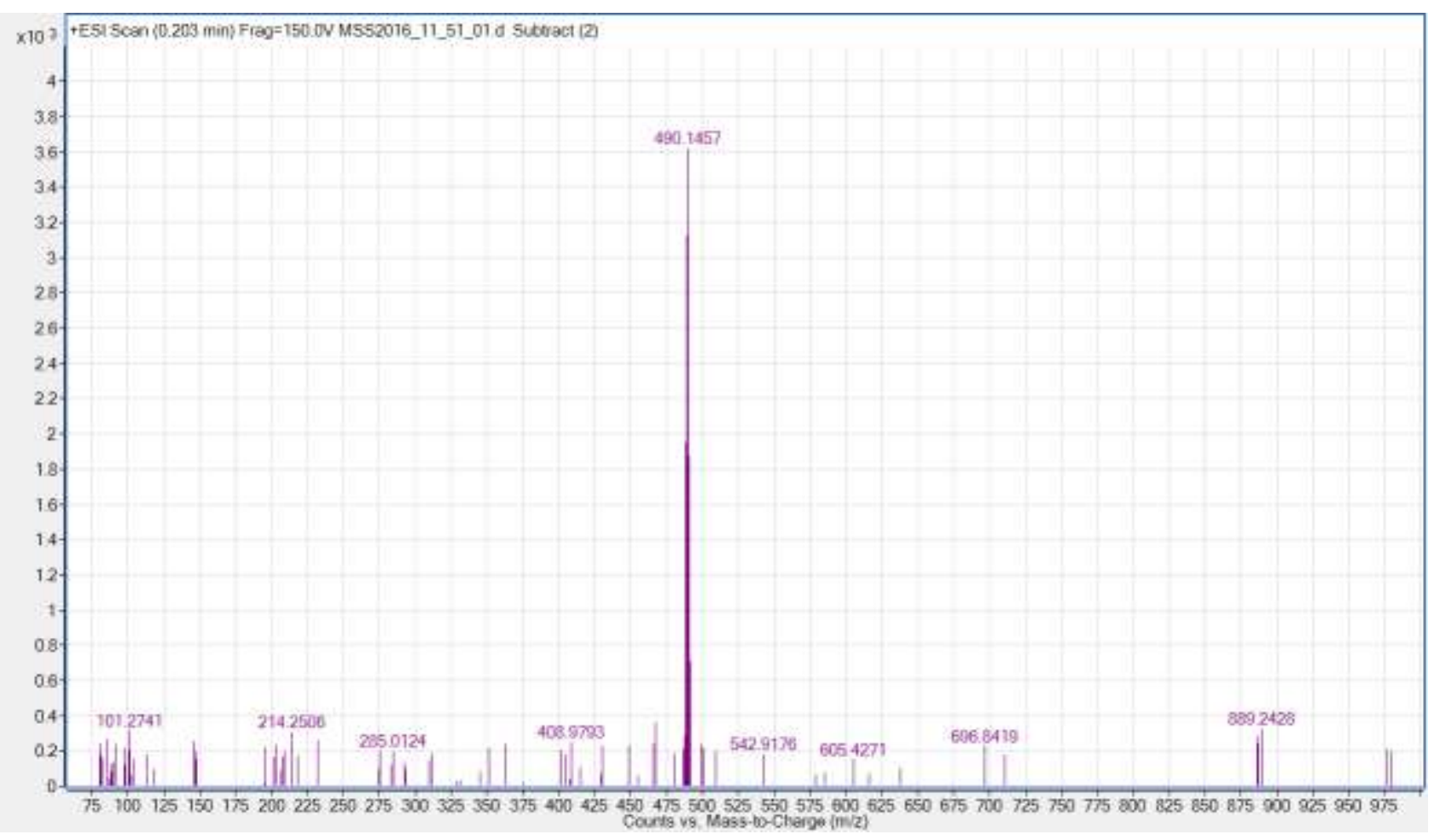

Figure S18 High resolution ESI mass spectrum for complex Os2. 


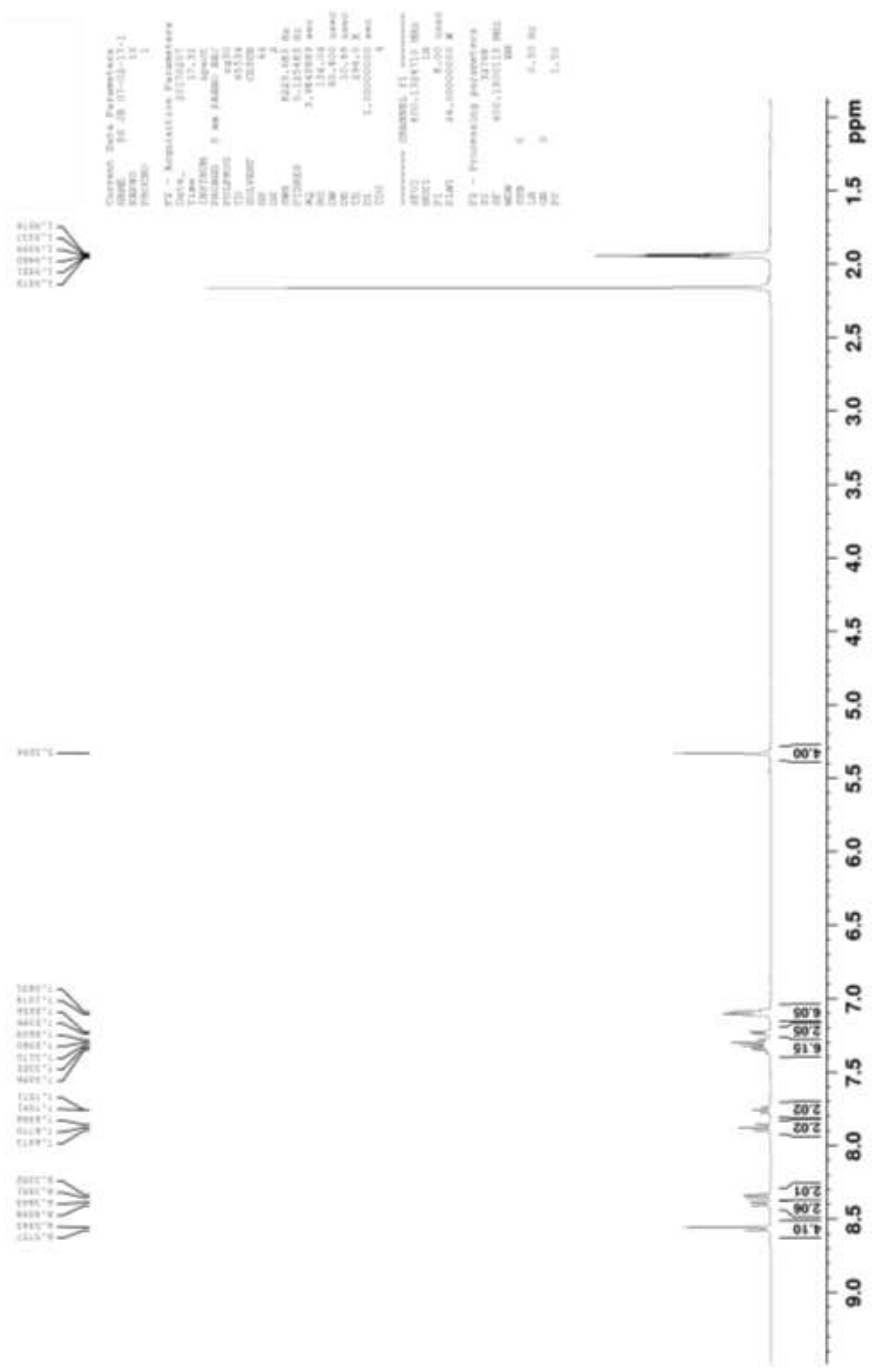

Figure S19 $\quad{ }^{1} \mathrm{H}$ NMR spectrum $\left(\mathrm{d}_{3}-\mathrm{MeCN}, 400 \mathrm{MHz}\right)$ of complex Os3. 


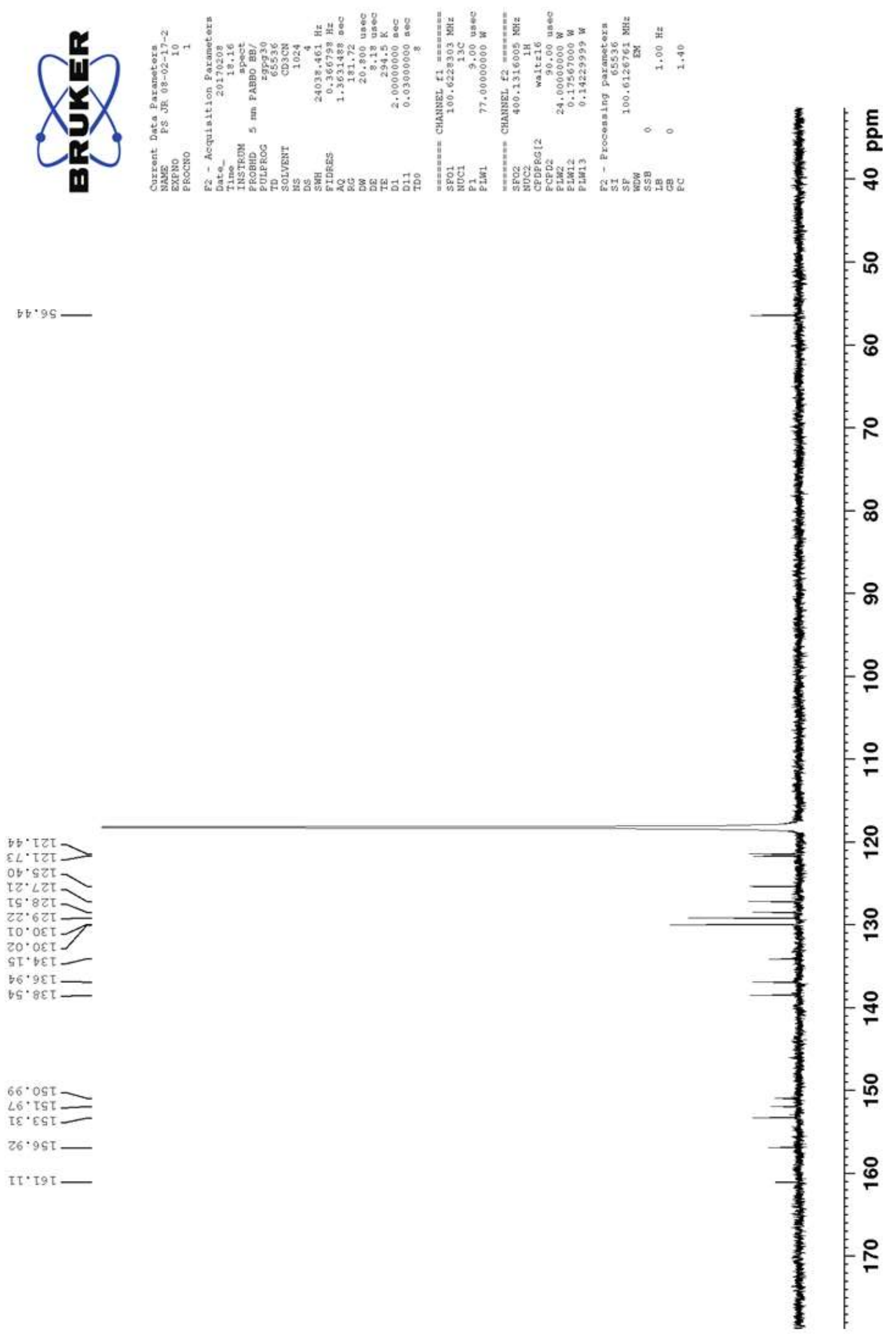

Figure S20 $\quad{ }^{13} \mathrm{C}$ NMR spectrum $\left(\mathrm{d}_{3}-\mathrm{MeCN}, 101 \mathrm{MHz}\right)$ of complex Os3. 


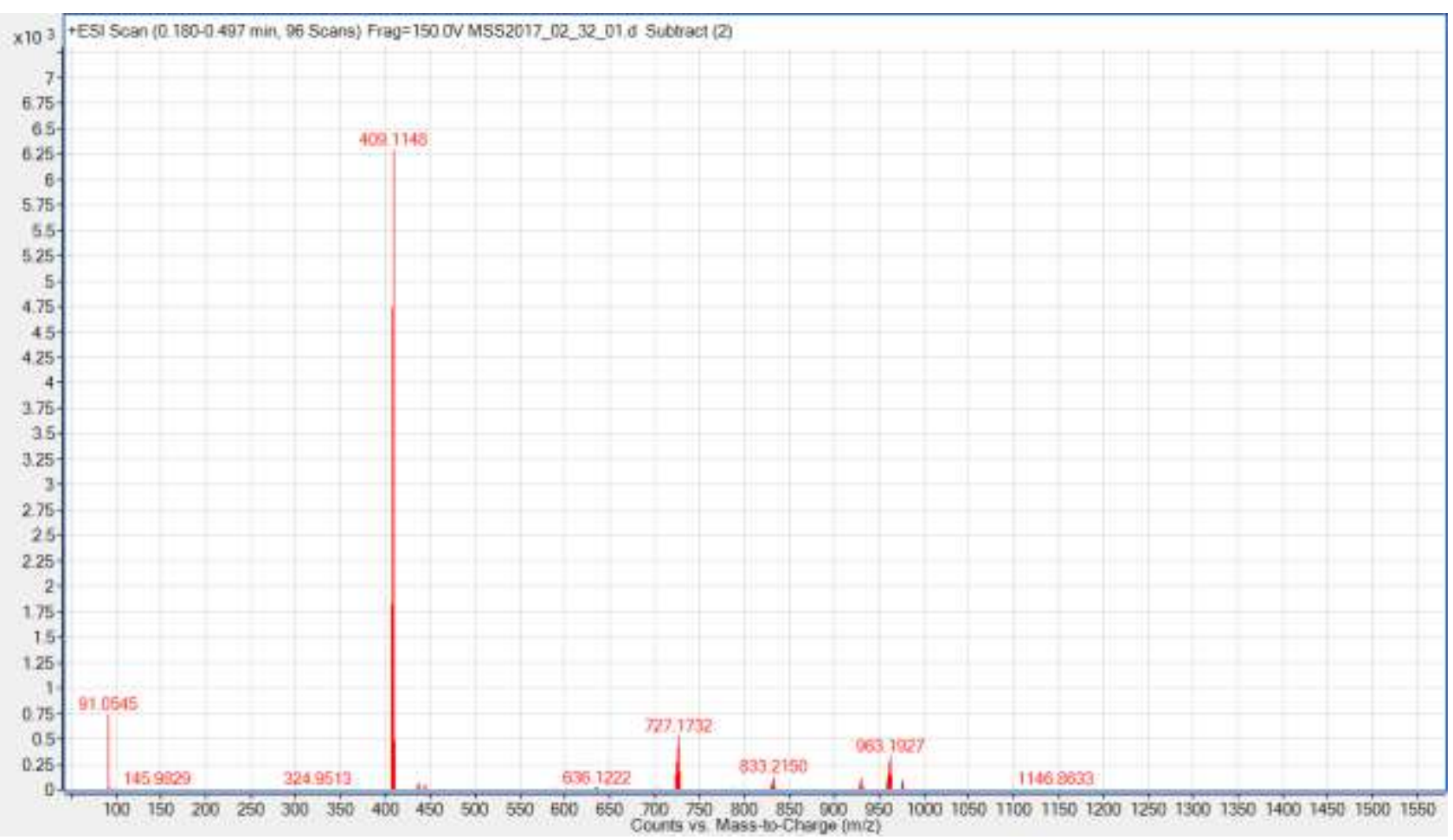

Figure S21 High resolution ESI mass spectrum for complex Os3. 


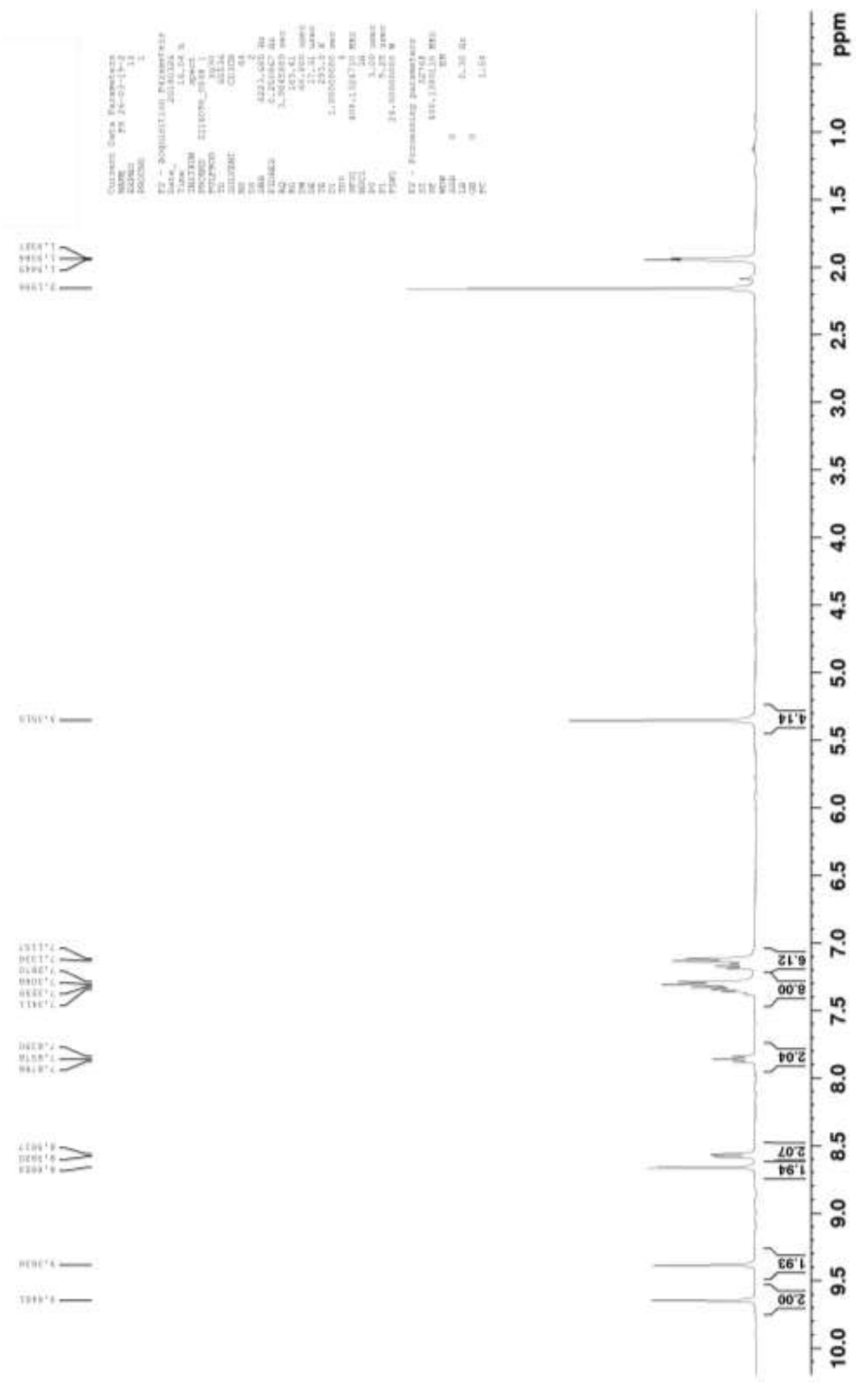

Figure S22 $\quad{ }^{1} \mathrm{H}$ NMR spectrum $\left(\mathrm{d}_{3}-\mathrm{MeCN}, 400 \mathrm{MHz}\right)$ of complex Os4. 


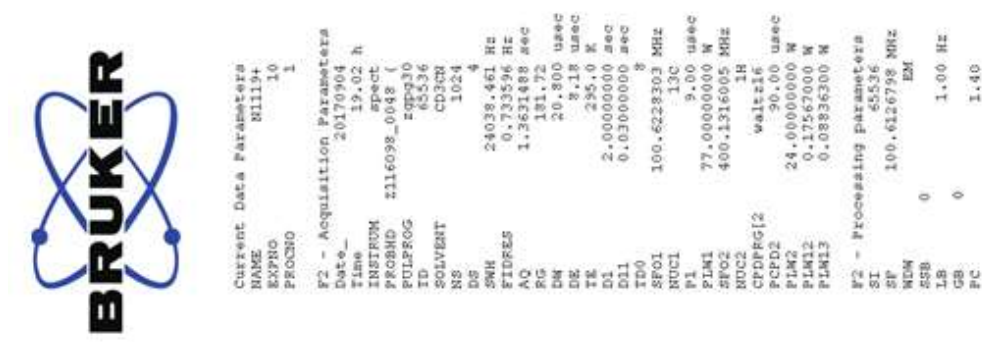

$69 \cdot 99$

$48^{\circ}$ SZI

$99^{\circ} \angle Z T$

$9 L^{\circ} 8 Z \tau$
$6 \varepsilon^{\circ} 6 \tau \tau$

Z0.0ET

OT. OET

$69 \cdot 6 \varepsilon \tau$

$96 . \tau b \mathrm{~T}$

$89.2 b \mathrm{I}=$

E9.

$90.6 \mathrm{VT}$

Z४.05T

$0 b^{*} \forall G T$

$6 \varsigma \cdot 8 \varsigma \tau$

틍

8

온

ஜ

ㅇ

음

을

สิ

옥

운

유

웅

Figure S23 $\quad{ }^{13} \mathrm{C}$ NMR spectrum $\left(\mathrm{d}_{3}-\mathrm{MeCN}, 101 \mathrm{MHz}\right)$ of complex Os4. 


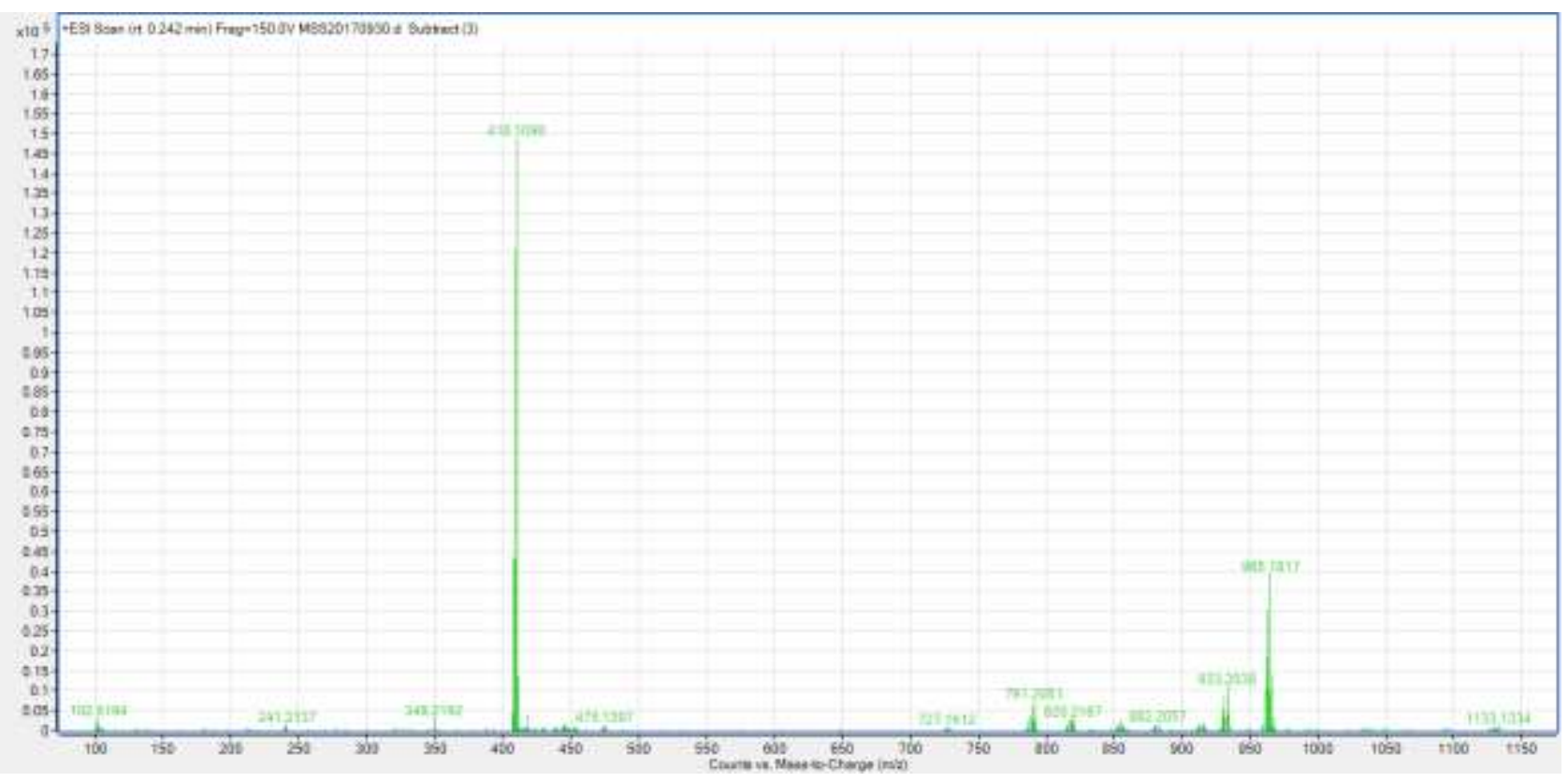

Figure S24 High resolution ESI mass spectrum for complex Os4. 

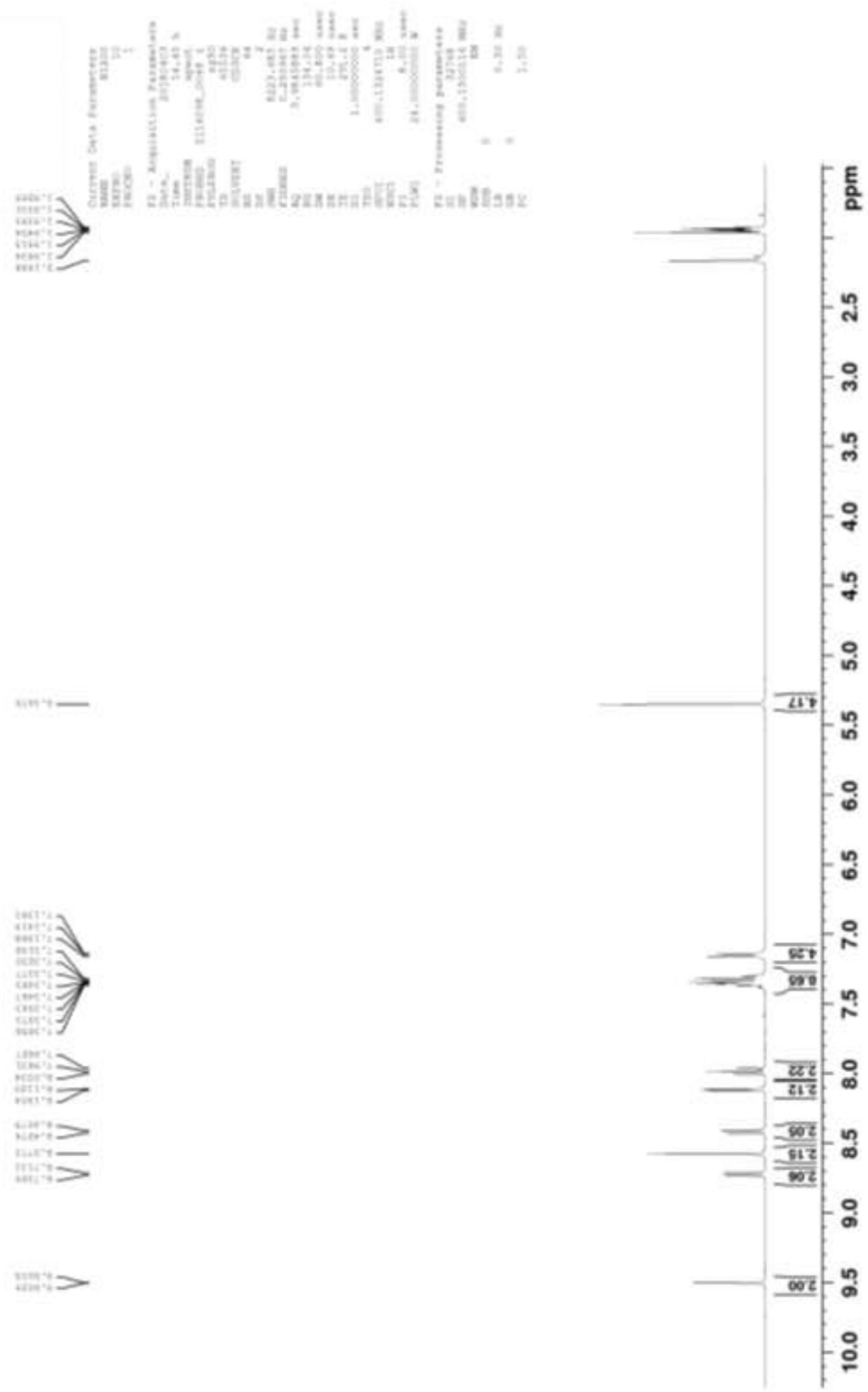

Figure S25 $\quad{ }^{1} \mathrm{H}$ NMR spectrum $\left(\mathrm{d}_{3}-\mathrm{MeCN}, 400 \mathrm{MHz}\right)$ of complex Os5. 


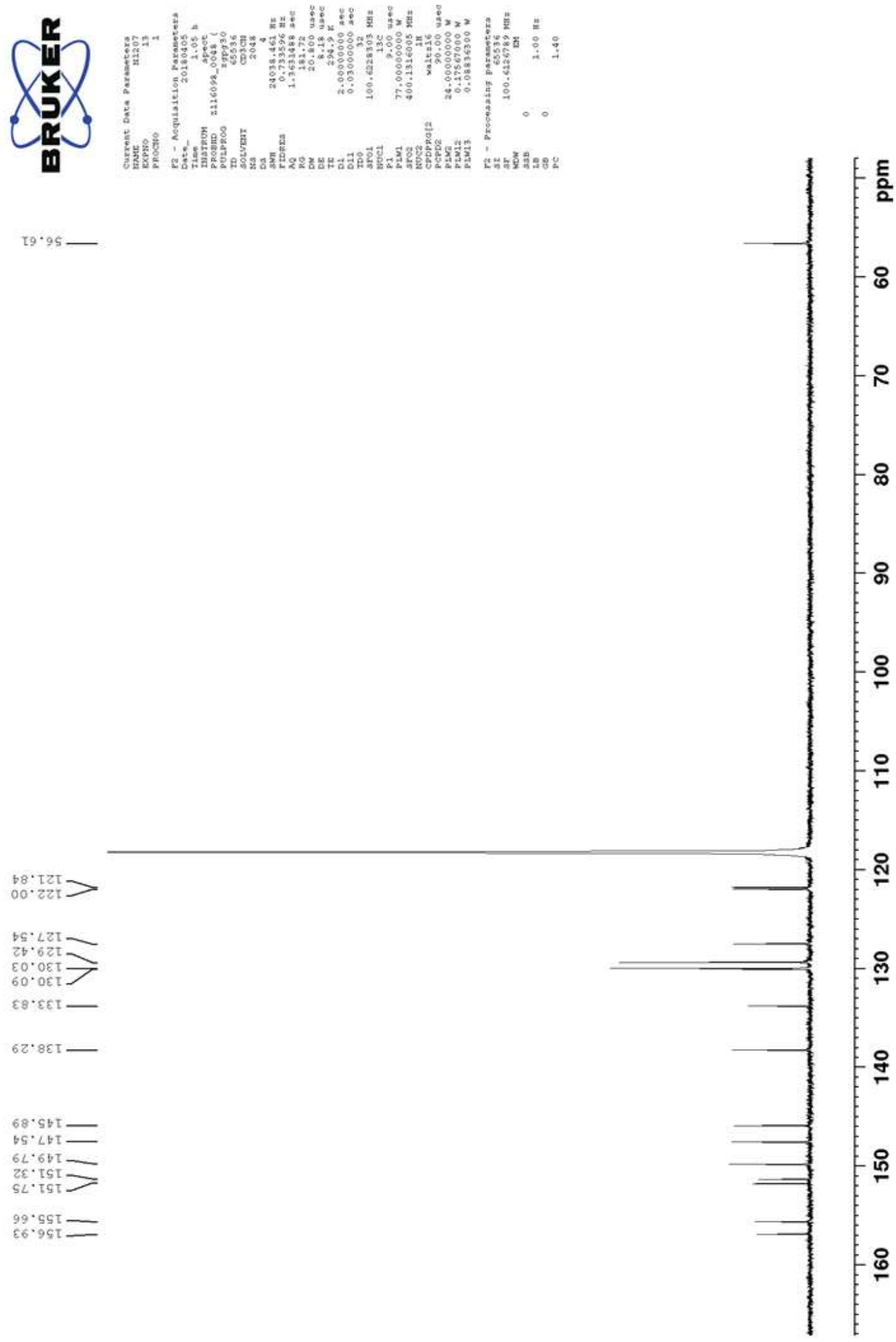

Figure S26 $\quad{ }^{13} \mathrm{C}$ NMR spectrum $\left(\mathrm{d}_{3}-\mathrm{MeCN}, 101 \mathrm{MHz}\right)$ of complex Os5. 


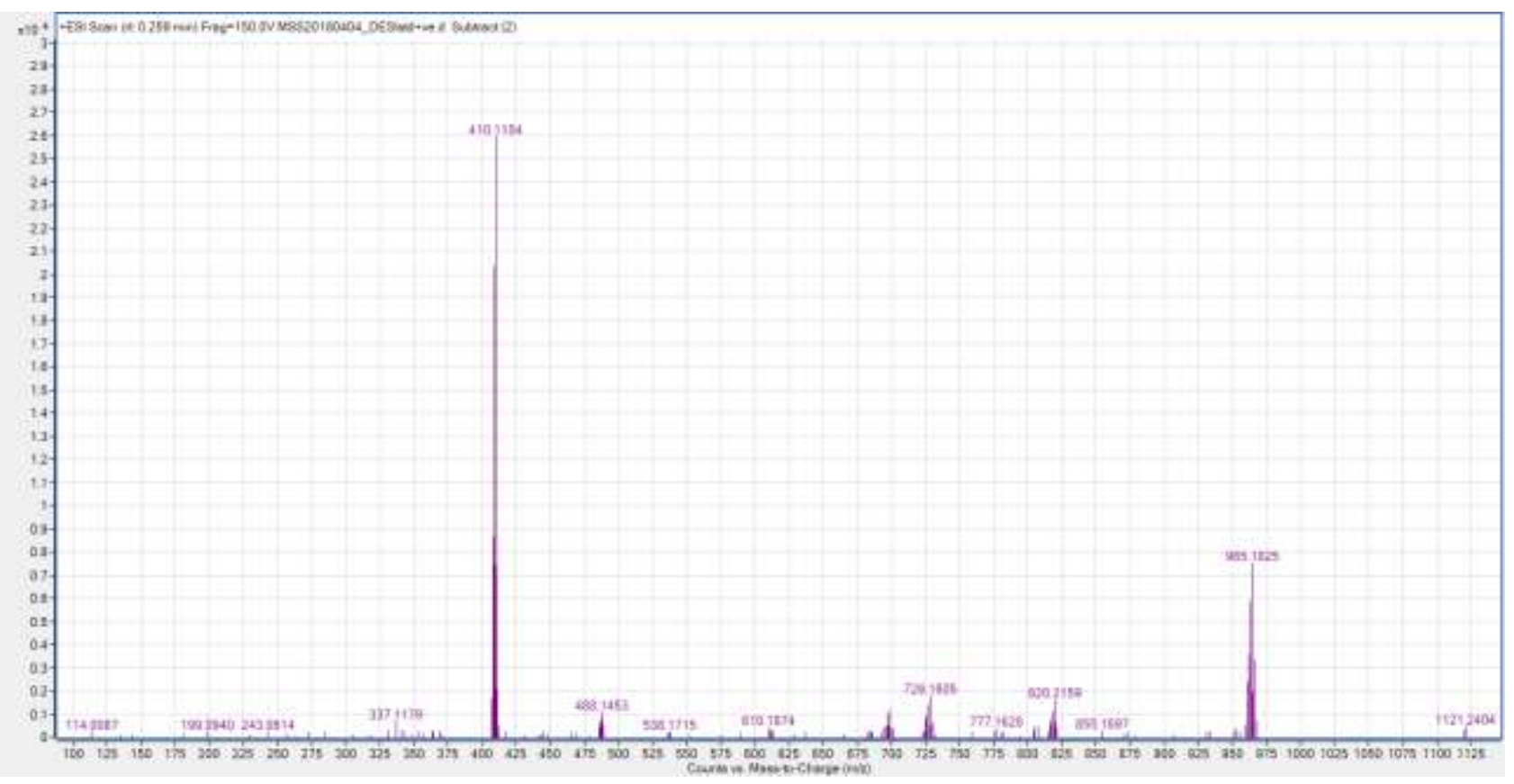

Figure S27 High resolution ESI mass spectrum for complex Os5 


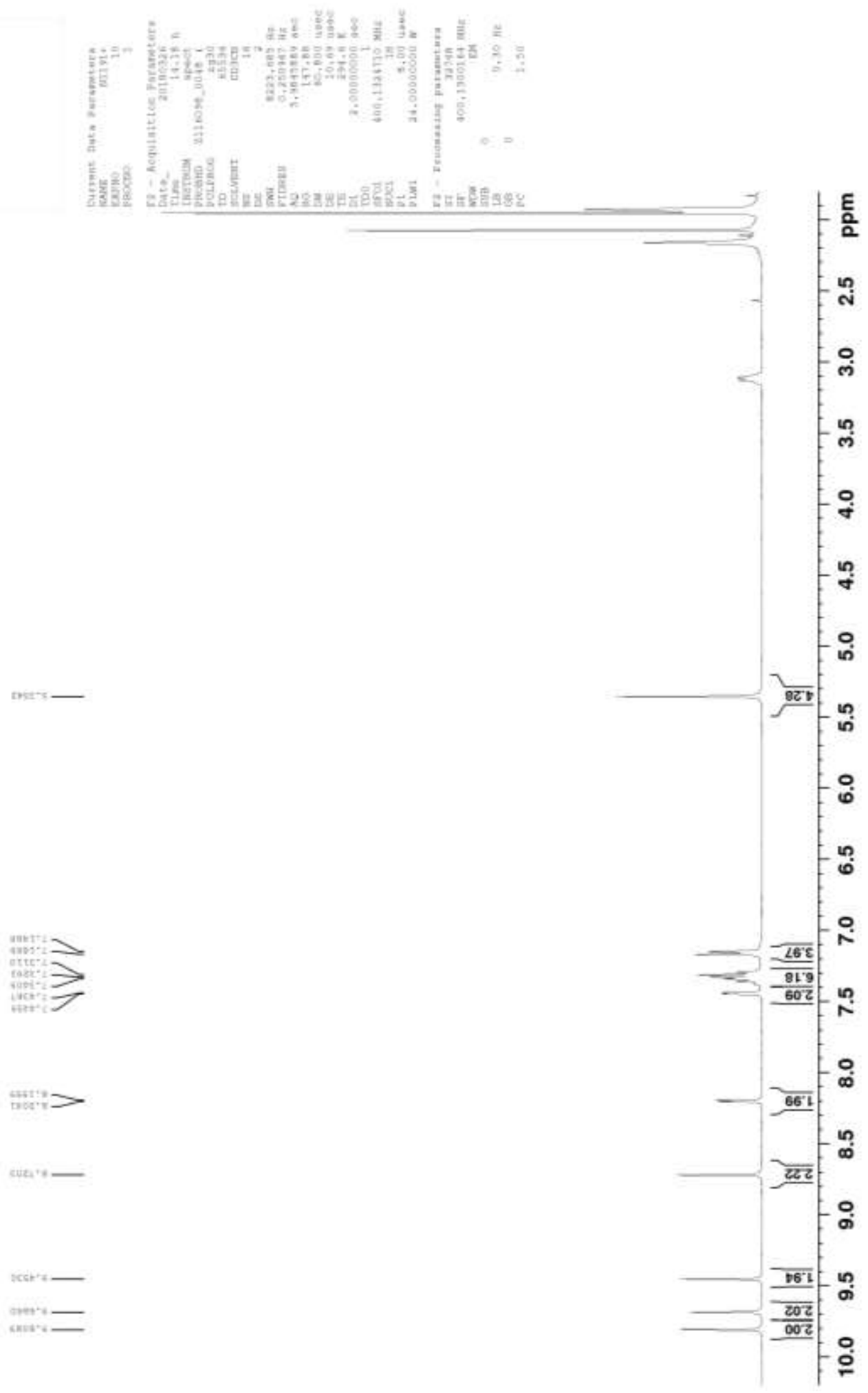

Figure S28 $\quad{ }^{1} \mathrm{H}$ NMR spectrum $\left(\mathrm{d}_{3}-\mathrm{MeCN}, 400 \mathrm{MHz}\right)$ of complex Os6. 


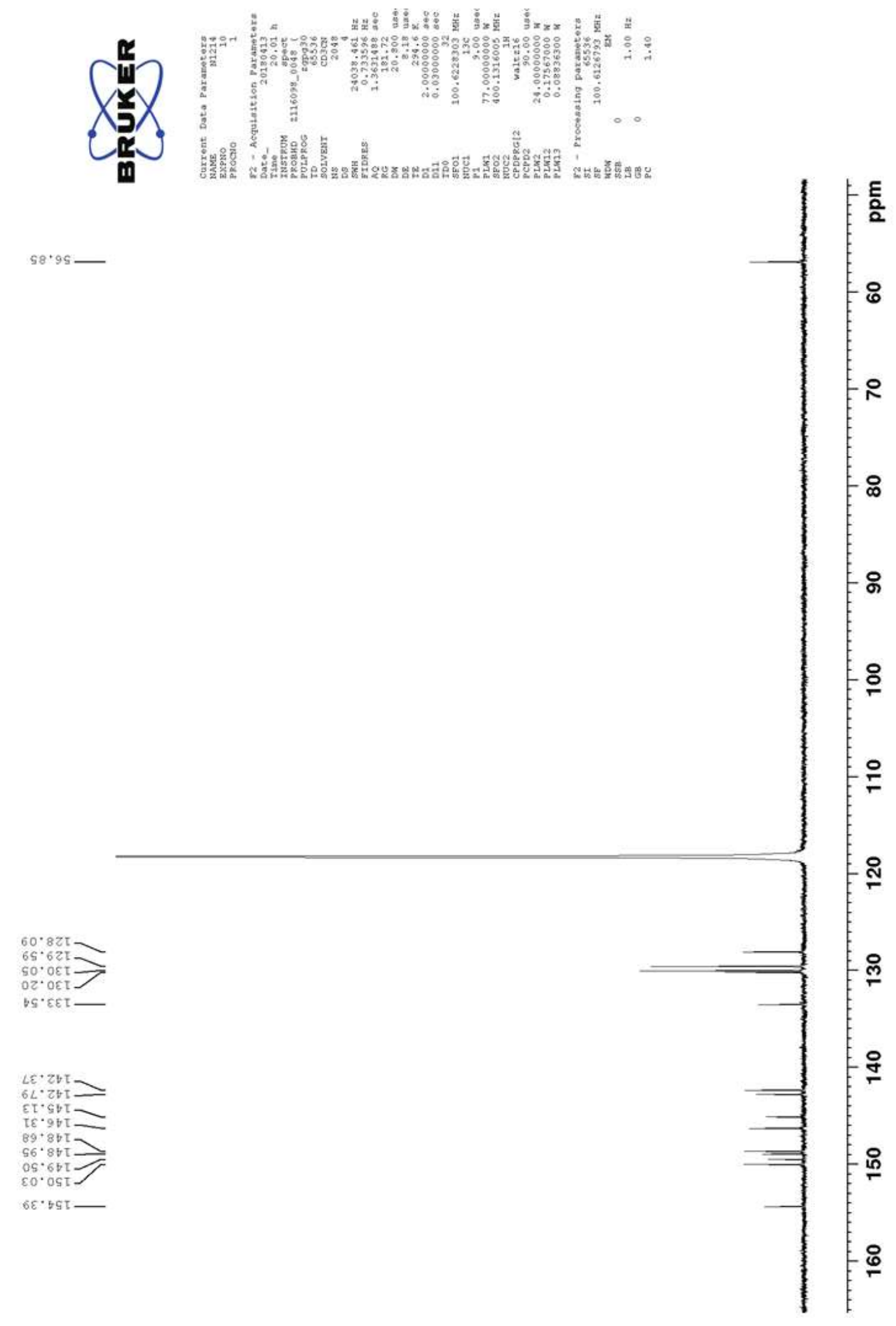

Figure S29 $\quad{ }^{13} \mathrm{C}$ NMR spectrum $\left(\mathrm{d}_{3}-\mathrm{MeCN}, 101 \mathrm{MHz}\right)$ of complex Os6. 


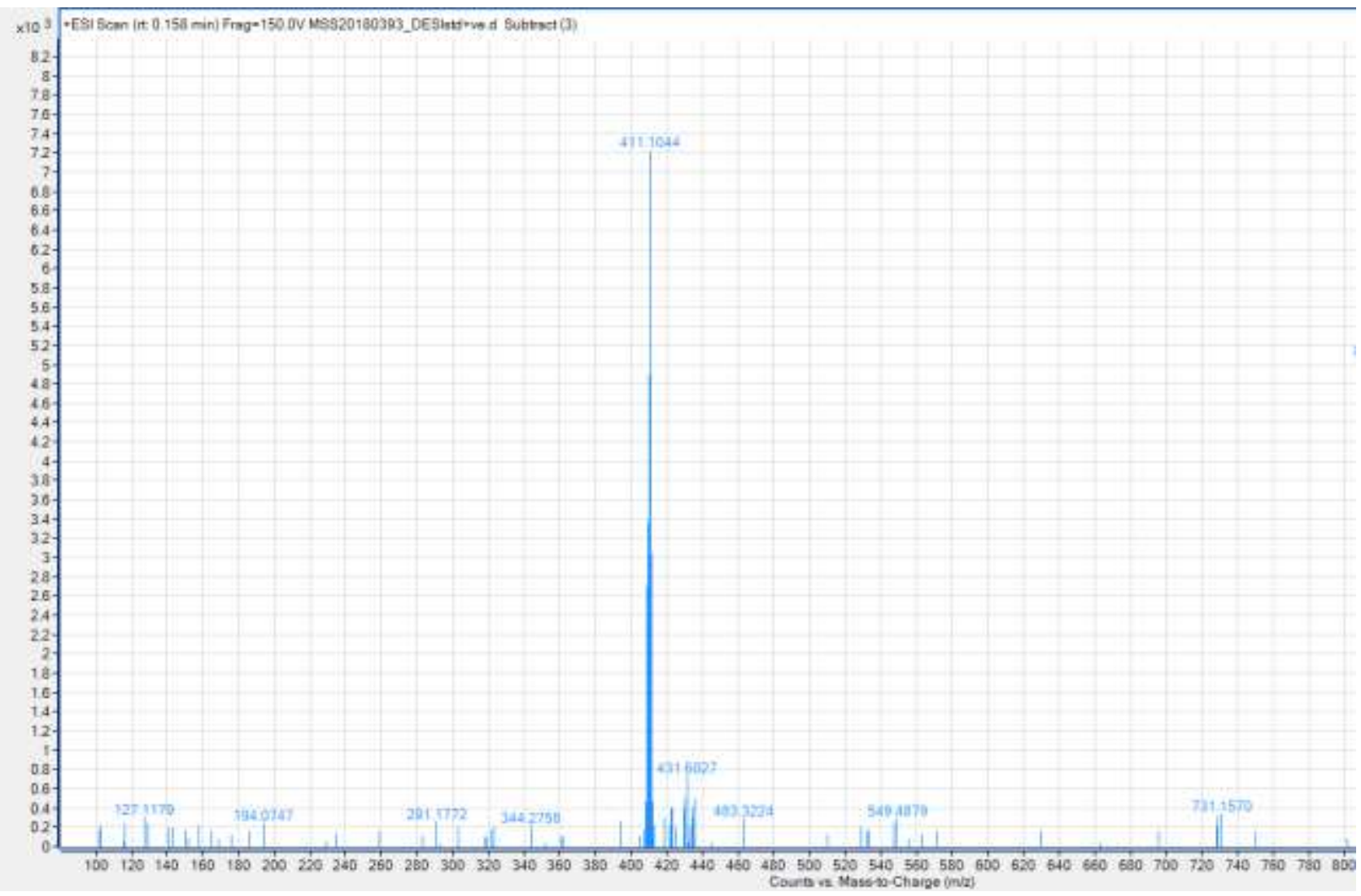

Figure S30 High resolution ESI mass spectrum for complex Os6. 


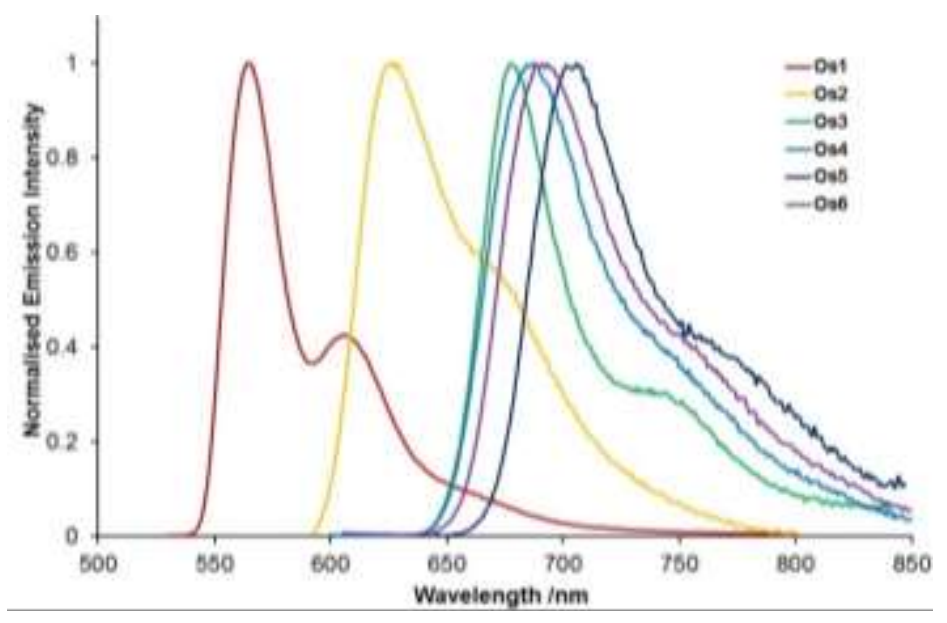

Figure S31. Low temperature $(77 \mathrm{~K})$ emission spectra recorded for Os1-Os6 in 4:1 EtOH/MeOH glass matricies.

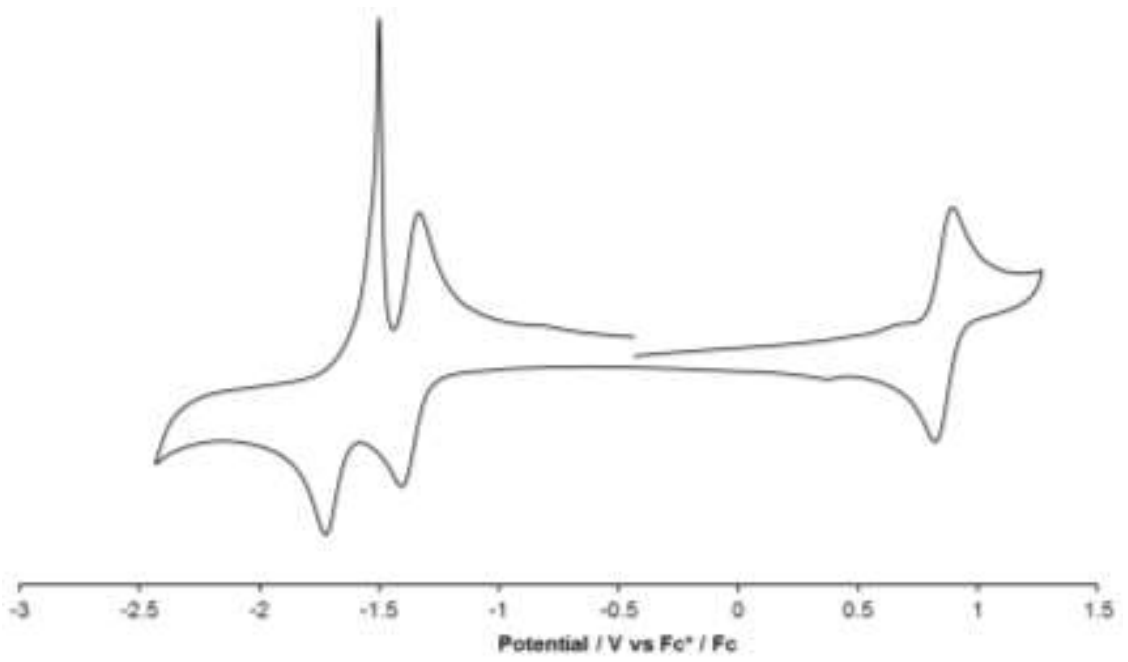

Figure S32. Additional electrochemical data for complex Os4 $\left(1.5 \mathrm{mmoldm}^{-3}\right)$ in $\mathrm{MeCN}$. Cyclic voltammogram recorded at $100 \mathrm{mVs}^{-1}$ with 0.2 moldm$^{-3} \mathrm{NBu}_{4} \mathrm{PF}_{6}$ as supporting electrolyte. The voltammogram shows the occurrence of a second ligand-based reduction process within the available electrochemical solvent window. The current spike in the return anodic wave is tentatively attributed to deposition or plating of the analyte upon the surface of the working electrode. The cathodic peak potential of the second reduction process is $-1.72 \mathrm{~V} v s \mathrm{Fc}^{+} / \mathrm{Fc}$. 


\section{Computational results}

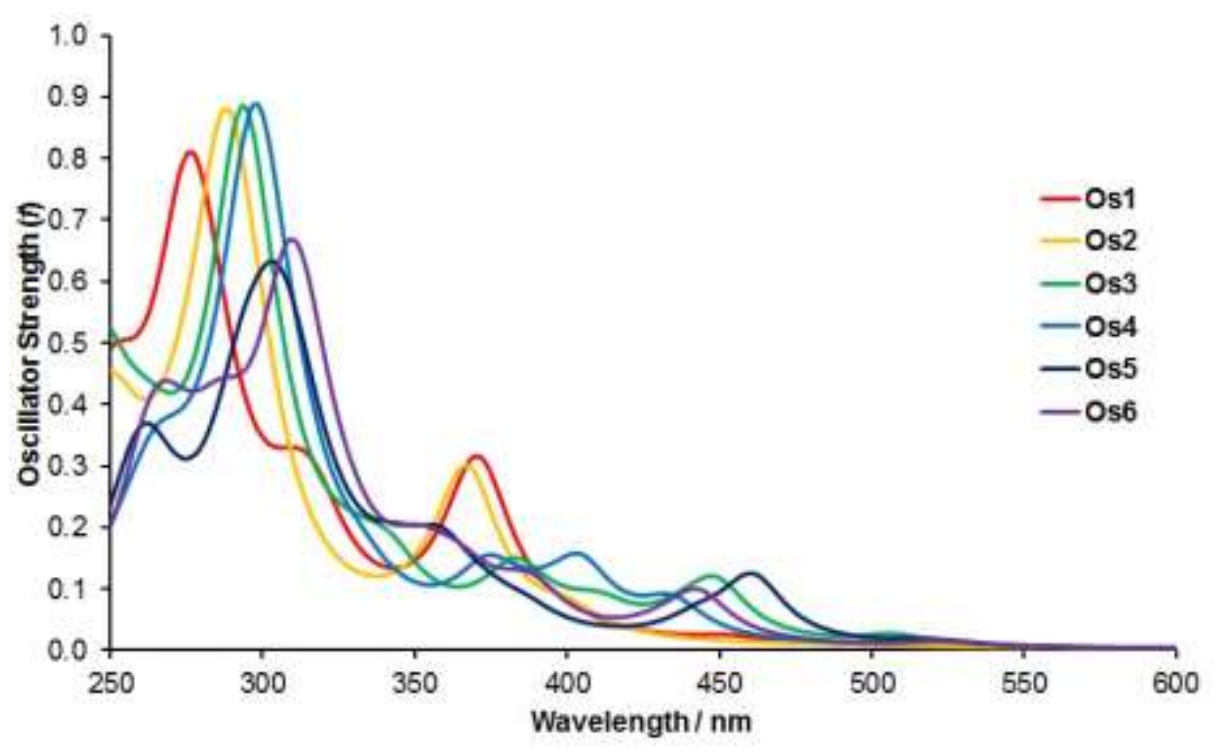

Figure S33. Calculated UV-visible absorption spectra of Os1-Os6 (spin-allowed singlet excitations only). 


\title{
Optimised ground state geometries
}

\author{
59
}

Geometry Os1

\begin{tabular}{|c|c|c|c|}
\hline Os & 0.00001779 & 0.00006954 & 0.00074860 \\
\hline $\mathrm{N}$ & 1.76644818 & 0.16956117 & 1.10723616 \\
\hline $\mathrm{N}$ & 2.54457565 & -0.66203095 & 1.74621125 \\
\hline $\mathrm{N}$ & 3.53441139 & 0.07512497 & 2.26433005 \\
\hline $\mathrm{C}$ & 3.39810172 & 1.38574443 & 1.95924768 \\
\hline $\mathrm{C}$ & 2.24760455 & 1.46175103 & 1.20432261 \\
\hline $\mathrm{C}$ & 1.48705568 & 2.51411326 & 0.54270318 \\
\hline $\mathrm{N}$ & 0.38830576 & 2.02035627 & -0.08272613 \\
\hline $\mathrm{C}$ & -0.45828150 & 2.83568114 & -0.76149195 \\
\hline $\mathrm{C}$ & -1.56439990 & 2.09183949 & -1.35118767 \\
\hline $\mathrm{C}$ & -2.67221744 & 2.39029304 & -2.11361163 \\
\hline $\mathrm{N}$ & -3.29119889 & 1.20415726 & -2.31666887 \\
\hline $\mathrm{N}$ & -2.63604858 & 0.19519776 & -1.72920790 \\
\hline $\mathrm{N}$ & -1.59530412 & 0.72487486 & -1.14615657 \\
\hline $\mathrm{C}$ & 1.77115952 & 3.87610164 & 0.50142215 \\
\hline $\mathrm{C}$ & 0.90848064 & 4.72210779 & -0.19278476 \\
\hline $\mathrm{C}$ & -0.21689957 & 4.20451900 & -0.83141651 \\
\hline $\mathrm{C}$ & 4.59443706 & -0.57657881 & 3.03666464 \\
\hline $\mathrm{C}$ & -4.51921412 & 0.93849510 & -3.06991934 \\
\hline $\mathrm{N}$ & -1.18006247 & -0.16398322 & 1.71938742 \\
\hline $\mathrm{N}$ & -1.64185376 & 0.67085285 & 2.61112280 \\
\hline $\mathrm{N}$ & -2.34465510 & -0.06338372 & 3.48207825 \\
\hline $\mathrm{C}$ & -2.33871812 & -1.37541830 & 3.15367403 \\
\hline $\mathrm{C}$ & -1.58177744 & -1.45542439 & 2.00478075 \\
\hline $\mathrm{C}$ & -1.14616192 & -2.51089757 & 1.09939110 \\
\hline $\mathrm{N}$ & -0.38761166 & -2.02032967 & 0.08639889 \\
\hline $\mathrm{C}$ & 0.11993486 & -2.83887753 & -0.86990784 \\
\hline $\mathrm{C}$ & 0.89937615 & -2.09811162 & -1.85388651 \\
\hline $\mathrm{C}$ & 1.61212660 & -2.40018824 & -2.99333755 \\
\hline $\mathrm{N}$ & 2.09754790 & -1.21518718 & -3.43086242 \\
\hline $\mathrm{N}$ & 1.72988684 & -0.20359686 & -2.63466567 \\
\hline $\mathrm{N}$ & 1.00795120 & -0.73040256 & -1.68338125 \\
\hline $\mathrm{C}$ & -1.42151712 & -3.87290911 & 1.18005996 \\
\hline $\mathrm{C}$ & -0.90511976 & -4.72227014 & 0.20348051 \\
\hline $\mathrm{C}$ & -0.12762643 & -4.20787908 & -0.83246740 \\
\hline $\mathrm{C}$ & -3.00514767 & 0.59130634 & 4.61347274 \\
\hline $\mathrm{C}$ & 2.92954695 & -0.95407333 & -4.60797620 \\
\hline $\mathrm{H}$ & 4.10162549 & 2.13198515 & 2.28994321 \\
\hline $\mathrm{H}$ & -3.05456620 & 3.31600229 & -2.51189648 \\
\hline $\mathrm{H}$ & 2.64785005 & 4.27153540 & 0.99989328 \\
\hline $\mathrm{H}$ & 1.11323643 & 5.78516357 & -0.23614387 \\
\hline $\mathrm{H}$ & -0.89188028 & 4.85627584 & -1.37273210 \\
\hline $\mathrm{H}$ & 5.24191905 & 0.19158046 & 3.45330265 \\
\hline $\mathrm{H}$ & 5.17118218 & -1.23151196 & 2.38496132 \\
\hline $\mathrm{H}$ & -4.72699007 & -0.12515320 & -2.99104785 \\
\hline $\mathrm{H}$ & -4.37812878 & 1.21046948 & -4.11561880 \\
\hline $\mathrm{H}$ & -2.85265900 & -2.11977029 & 3.73929977 \\
\hline $\mathrm{H}$ & 1.80500606 & -3.32763962 & -3.50751910 \\
\hline $\mathrm{H}$ & -2.02668330 & -4.26588318 & 1.98799423 \\
\hline $\mathrm{H}$ & -1.10870082 & -5.78543105 & 0.24958245 \\
\hline $\mathrm{H}$ & 0.27691124 & -4.86220186 & -1.59510050 \\
\hline $\mathrm{H}$ & -3.75665165 & 1.28683294 & 4.24286248 \\
\hline $\mathrm{H}$ & -3.47999161 & -0.17172043 & 5.22595619 \\
\hline $\mathrm{H}$ & 3.11310423 & 0.11622175 & -4.64708320 \\
\hline $\mathrm{H}$ & 2.40509276 & -1.27174298 & -5.50862025 \\
\hline $\mathrm{H}$ & 4.14631538 & -1.15625007 & 3.84154512 \\
\hline $\mathrm{H}$ & -5.34411643 & 1.50828536 & -2.64342163 \\
\hline $\mathrm{H}$ & -2.26306793 & 1.12717249 & 5.20277201 \\
\hline $\mathrm{H}$ & 3.87529628 & -1.48770417 & -4.51813150 \\
\hline
\end{tabular}


Geometry Os2

\begin{tabular}{|c|c|c|c|}
\hline Os & 0.00040509 & -0.00012941 & -0.00051545 \\
\hline $\mathrm{N}$ & 1.59535853 & -0.33506732 & 1.31866070 \\
\hline $\mathrm{N}$ & 2.45172372 & 0.42354879 & 1.94677387 \\
\hline $\mathrm{N}$ & 3.23763709 & -0.40117618 & 2.64818299 \\
\hline $\mathrm{C}$ & 2.89023740 & -1.69674152 & 2.47590934 \\
\hline $\mathrm{C}$ & 1.81864923 & -1.66842240 & 1.60934944 \\
\hline $\mathrm{C}$ & 0.93743954 & -2.63661754 & 0.97593378 \\
\hline $\mathrm{N}$ & 0.01688973 & -2.03613995 & 0.18190669 \\
\hline $\mathrm{C}$ & -0.89179896 & -2.78399414 & -0.49157987 \\
\hline $\mathrm{C}$ & -1.78953875 & -1.95895434 & -1.28492266 \\
\hline $\mathrm{C}$ & -2.85290519 & -2.16064618 & -2.13765590 \\
\hline $\mathrm{N}$ & -3.21939800 & -0.92255288 & -2.54181649 \\
\hline $\mathrm{N}$ & -2.45429332 & 0.02756470 & -1.99232887 \\
\hline $\mathrm{N}$ & -1.58998499 & -0.59120101 & -1.23562545 \\
\hline $\mathrm{C}$ & 0.92651430 & -4.02840065 & 1.08155840 \\
\hline $\mathrm{N}$ & 0.04095332 & -4.77882692 & 0.42709217 \\
\hline $\mathrm{C}$ & -0.85676573 & -4.17183108 & -0.34868574 \\
\hline $\mathrm{C}$ & 4.30885733 & 0.15303408 & 3.48144436 \\
\hline $\mathrm{C}$ & -4.32780598 & -0.54709236 & -3.42499907 \\
\hline $\mathrm{N}$ & 1.27963356 & 0.32824426 & -1.62889587 \\
\hline $\mathrm{N}$ & 1.97939674 & -0.43400089 & -2.42440200 \\
\hline $\mathrm{N}$ & 2.60119185 & 0.38739541 & -3.27794059 \\
\hline $\mathrm{C}$ & 2.30385696 & 1.68441327 & -3.03670088 \\
\hline $\mathrm{C}$ & 1.44141892 & 1.66061029 & -1.96162028 \\
\hline $\mathrm{C}$ & 0.71851461 & 2.63245915 & -1.15656023 \\
\hline $\mathrm{N}$ & -0.01447149 & 2.03594692 & -0.18428848 \\
\hline $\mathrm{C}$ & -0.75683365 & 2.78744168 & 0.66562190 \\
\hline $\mathrm{C}$ & -1.46802146 & 1.96630421 & 1.63284036 \\
\hline $\mathrm{C}$ & -2.33855300 & 2.17146018 & 2.68099531 \\
\hline $\mathrm{N}$ & -2.62299068 & 0.93441952 & 3.14924758 \\
\hline $\mathrm{N}$ & -1.98318985 & -0.01795498 & 2.46096639 \\
\hline $\mathrm{N}$ & -1.28743926 & 0.59793175 & 1.54495005 \\
\hline $\mathrm{C}$ & 0.69017474 & 4.02420827 & -1.25943566 \\
\hline $\mathrm{N}$ & -0.03342617 & 4.77826324 & -0.43262122 \\
\hline $\mathrm{C}$ & -0.74771530 & 4.17506684 & 0.51708849 \\
\hline $\mathrm{C}$ & 3.46932064 & -0.17141896 & -4.31866655 \\
\hline $\mathrm{C}$ & -3.46673545 & 0.56769211 & 4.29079516 \\
\hline $\mathrm{H}$ & 3.41026981 & -2.50561168 & 2.96246189 \\
\hline $\mathrm{H}$ & -3.35665566 & -3.05198252 & -2.47480188 \\
\hline $\mathrm{H}$ & 1.64453989 & -4.55155449 & 1.70539013 \\
\hline $\mathrm{H}$ & -1.56469590 & -4.80972778 & -0.86858208 \\
\hline $\mathrm{H}$ & 4.87877018 & -0.67079492 & 3.90472774 \\
\hline $\mathrm{H}$ & 3.87455231 & 0.75122270 & 4.28106425 \\
\hline $\mathrm{H}$ & -4.14689695 & 0.46677552 & -3.77193359 \\
\hline $\mathrm{H}$ & -5.26959082 & -0.58916132 & -2.87785335 \\
\hline $\mathrm{H}$ & 2.71176323 & 2.49107519 & -3.62352412 \\
\hline $\mathrm{H}$ & -2.76122004 & 3.06447126 & 3.11194867 \\
\hline $\mathrm{H}$ & 1.26097166 & 4.54439735 & -2.02236068 \\
\hline $\mathrm{H}$ & -1.32645819 & 4.81592209 & 1.17484986 \\
\hline $\mathrm{H}$ & 2.87515426 & -0.78022626 & -4.99843661 \\
\hline $\mathrm{H}$ & 3.92798226 & 0.65011882 & -4.86398326 \\
\hline $\mathrm{H}$ & -3.74012764 & -0.47830318 & 4.18011912 \\
\hline $\mathrm{H}$ & -4.36270047 & 1.18589525 & 4.28805926 \\
\hline $\mathrm{H}$ & 4.95792049 & 0.77219184 & 2.86501716 \\
\hline $\mathrm{H}$ & -4.35804129 & -1.22810949 & -4.27378520 \\
\hline $\mathrm{H}$ & 4.24119699 & -0.78098653 & -3.85238908 \\
\hline $\mathrm{H}$ & -2.91647378 & 0.71061007 & 5.22081312 \\
\hline
\end{tabular}


59

Geometry Os3

\begin{tabular}{|c|c|c|c|}
\hline Os & -0.08598066 & 0.00097079 & -0.00427356 \\
\hline $\mathrm{N}$ & -0.08475835 & 1.91956266 & 0.69281048 \\
\hline $\mathrm{N}$ & 1.28328695 & -0.10184536 & 1.57619496 \\
\hline $\mathrm{N}$ & -1.61053816 & -0.92982605 & 1.13074259 \\
\hline $\mathrm{N}$ & 1.43933804 & 0.08735750 & -1.43899383 \\
\hline $\mathrm{N}$ & -0.03475530 & -1.91777144 & -0.69904858 \\
\hline $\mathrm{N}$ & -1.47711218 & 0.94834975 & -1.28852447 \\
\hline $\mathrm{C}$ & -0.88257995 & -2.83102376 & -0.16447321 \\
\hline $\mathrm{C}$ & 0.07833424 & -4.52136220 & -1.58138436 \\
\hline $\mathrm{C}$ & -3.37731878 & -1.05392365 & 2.75332958 \\
\hline $\mathrm{C}$ & 1.68175717 & -1.12173080 & -2.06409424 \\
\hline $\mathrm{C}$ & -0.83996583 & -4.15536672 & -0.60041230 \\
\hline $\mathrm{C}$ & -1.77799413 & -2.26736719 & 0.86238781 \\
\hline $\mathrm{C}$ & 0.94543156 & -3.57093666 & -2.11727695 \\
\hline $\mathrm{C}$ & -2.40147577 & -0.35590366 & 2.05741548 \\
\hline $\mathrm{C}$ & -2.74401677 & -3.01361608 & 1.53444304 \\
\hline $\mathrm{C}$ & 0.87118438 & -2.26161579 & -1.65471635 \\
\hline $\mathrm{C}$ & -3.55289510 & -2.40851980 & 2.48840387 \\
\hline $\mathrm{C}$ & 2.69660604 & -0.88933366 & -2.96697247 \\
\hline $\mathrm{C}$ & 4.01538428 & 1.19981454 & -3.54283772 \\
\hline $\mathrm{C}$ & -0.86309872 & 2.84209408 & 0.07495270 \\
\hline $\mathrm{C}$ & 0.72368006 & 2.25332218 & 1.73557415 \\
\hline $\mathrm{C}$ & 1.47469519 & 1.10408554 & 2.22397386 \\
\hline $\mathrm{C}$ & -1.65583731 & 2.28781916 & -1.03788582 \\
\hline $\mathrm{C}$ & -0.84956255 & 4.16616155 & 0.51329639 \\
\hline $\mathrm{C}$ & -0.03138391 & 4.52207354 & 1.58272508 \\
\hline $\mathrm{C}$ & -2.17642258 & 0.38270214 & -2.29082562 \\
\hline $\mathrm{C}$ & 0.76575373 & 3.56226242 & 2.20362455 \\
\hline $\mathrm{C}$ & -2.54004657 & 3.04449302 & -1.80422726 \\
\hline $\mathrm{C}$ & 2.39087835 & 0.85901164 & 3.22467939 \\
\hline $\mathrm{C}$ & 3.60979635 & -1.25301632 & 3.93063098 \\
\hline $\mathrm{C}$ & -3.06871090 & 1.09117393 & -3.08189411 \\
\hline $\mathrm{C}$ & -3.25471710 & 2.44790139 & -2.83565303 \\
\hline $\mathrm{N}$ & 2.99629429 & 0.42322139 & -2.83120817 \\
\hline $\mathrm{N}$ & 2.23356511 & 1.01367852 & -1.90312733 \\
\hline $\mathrm{N}$ & 2.01577430 & -1.03820899 & 2.11695553 \\
\hline $\mathrm{N}$ & 2.68751054 & -0.45611312 & 3.11745189 \\
\hline $\mathrm{H}$ & 3.86622020 & 1.10246486 & -4.61743685 \\
\hline $\mathrm{H}$ & 3.89965207 & 2.23922133 & -3.24794738 \\
\hline $\mathrm{H}$ & 5.00919897 & 0.84616656 & -3.26921129 \\
\hline $\mathrm{H}$ & 4.40683805 & -1.64168840 & 3.29875237 \\
\hline $\mathrm{H}$ & 4.03076792 & -0.61365879 & 4.70311639 \\
\hline $\mathrm{H}$ & 3.06551821 & -2.07636532 & 4.38999288 \\
\hline $\mathrm{H}$ & 0.12034314 & -5.54768323 & -1.92583782 \\
\hline $\mathrm{H}$ & -3.98436792 & -0.53782305 & 3.48699463 \\
\hline $\mathrm{H}$ & -1.50746235 & -4.89824756 & -0.18364805 \\
\hline $\mathrm{H}$ & 1.66644654 & -3.84738151 & -2.87710084 \\
\hline $\mathrm{H}$ & -2.23378633 & 0.69872599 & 2.23452842 \\
\hline $\mathrm{H}$ & -2.86588921 & -4.06615462 & 1.31305534 \\
\hline $\mathrm{H}$ & -4.30583322 & -2.98467064 & 3.01318033 \\
\hline $\mathrm{H}$ & 3.20873382 & -1.52807510 & -3.66812626 \\
\hline $\mathrm{H}$ & -1.46238693 & 4.91656175 & 0.03118648 \\
\hline $\mathrm{H}$ & -0.01263957 & 5.54811415 & 1.93007266 \\
\hline $\mathrm{H}$ & -2.00345891 & -0.67388716 & -2.45054471 \\
\hline $\mathrm{H}$ & 1.40904361 & 3.83122647 & 3.03275761 \\
\hline $\mathrm{H}$ & -2.67175907 & 4.09852497 & -1.59600783 \\
\hline $\mathrm{H}$ & 2.83631694 & 1.49214823 & 3.97435893 \\
\hline $\mathrm{H}$ & -3.60406673 & 0.58143092 & -3.87353537 \\
\hline $\mathrm{H}$ & -3.94377009 & 3.03222477 & -3.43401032 \\
\hline
\end{tabular}


Geometry Os4

\begin{tabular}{|c|c|c|c|}
\hline Os & -0.09164259 & 0.00100555 & -0.00540365 \\
\hline $\mathrm{N}$ & -0.08480625 & 1.92209171 & 0.64140408 \\
\hline $\mathrm{N}$ & 1.27209974 & -0.05993357 & 1.58805917 \\
\hline $\mathrm{N}$ & -1.62613125 & -0.91644220 & 1.14287578 \\
\hline $\mathrm{N}$ & 1.44356572 & 0.04614705 & -1.43796549 \\
\hline $\mathrm{N}$ & -0.03168095 & -1.92056099 & -0.64777525 \\
\hline $\mathrm{N}$ & -1.47892488 & 0.93440454 & -1.31760999 \\
\hline $\mathrm{C}$ & -0.87311346 & -2.83021887 & -0.10212282 \\
\hline $\mathrm{N}$ & 0.09569841 & -4.54731898 & -1.45822522 \\
\hline $\mathrm{C}$ & -3.40409751 & -1.02301826 & 2.75351356 \\
\hline $\mathrm{C}$ & 1.69336287 & -1.17893544 & -2.02935820 \\
\hline $\mathrm{C}$ & -0.78273661 & -4.15709475 & -0.53591554 \\
\hline $\mathrm{C}$ & -1.78188360 & -2.26157262 & 0.90351041 \\
\hline $\mathrm{C}$ & 0.91762774 & -3.63521035 & -1.97811574 \\
\hline $\mathrm{C}$ & -2.42884960 & -0.32984570 & 2.05057600 \\
\hline $\mathrm{C}$ & -2.74541656 & -3.00422443 & 1.58234475 \\
\hline $\mathrm{C}$ & 0.87715155 & -2.29806079 & -1.58611508 \\
\hline $\mathrm{C}$ & -3.56637383 & -2.38455723 & 2.51699383 \\
\hline $\mathrm{C}$ & 2.71484996 & -0.96809528 & -2.93030949 \\
\hline $\mathrm{C}$ & 4.02706972 & 1.11091410 & -3.55805143 \\
\hline $\mathrm{C}$ & -0.85077061 & 2.84031789 & 0.00634288 \\
\hline $\mathrm{C}$ & 0.71658920 & 2.28948186 & 1.67661279 \\
\hline $\mathrm{C}$ & 1.46623675 & 1.16166699 & 2.20604858 \\
\hline $\mathrm{C}$ & -1.64652910 & 2.28107578 & -1.09580668 \\
\hline $\mathrm{C}$ & -0.79697243 & 4.16580066 & 0.45003487 \\
\hline $\mathrm{N}$ & -0.02396963 & 4.54639122 & 1.46612690 \\
\hline $\mathrm{C}$ & -2.18077609 & 0.35620551 & -2.31028516 \\
\hline $\mathrm{C}$ & 0.72546953 & 3.62598504 & 2.07336768 \\
\hline $\mathrm{C}$ & -2.51962209 & 3.03382970 & -1.87799939 \\
\hline $\mathrm{C}$ & 2.38028103 & 0.93883320 & 3.21418539 \\
\hline $\mathrm{C}$ & 3.58602666 & -1.16078878 & 3.97919621 \\
\hline $\mathrm{C}$ & -3.06331287 & 1.05954747 & -3.11786069 \\
\hline $\mathrm{C}$ & -3.23658674 & 2.42280028 & -2.89982916 \\
\hline $\mathrm{N}$ & 3.01006425 & 0.34824484 & -2.82565576 \\
\hline $\mathrm{N}$ & 2.23899847 & 0.96114801 & -1.92033292 \\
\hline $\mathrm{N}$ & 2.00034883 & -0.98415218 & 2.15315149 \\
\hline $\mathrm{N}$ & 2.67230155 & -0.37938651 & 3.13919821 \\
\hline $\mathrm{H}$ & 3.83852713 & 1.03940531 & -4.62853194 \\
\hline $\mathrm{H}$ & 3.95176400 & 2.14669379 & -3.23854546 \\
\hline $\mathrm{H}$ & 5.01769132 & 0.72212414 & -3.32457014 \\
\hline $\mathrm{H}$ & 4.35373891 & -1.61153008 & 3.35272508 \\
\hline $\mathrm{H}$ & 4.04603334 & -0.49231709 & 4.70314471 \\
\hline $\mathrm{H}$ & 3.02519212 & -1.93701791 & 4.49713492 \\
\hline $\mathrm{H}$ & -4.02128901 & -0.49672144 & 3.47129350 \\
\hline $\mathrm{H}$ & -1.42942522 & -4.92948246 & -0.13369301 \\
\hline $\mathrm{H}$ & 1.62553652 & -3.98339364 & -2.72394891 \\
\hline $\mathrm{H}$ & -2.27372907 & 0.72977555 & 2.20838566 \\
\hline $\mathrm{H}$ & -2.85463966 & -4.06243519 & 1.38099557 \\
\hline $\mathrm{H}$ & -4.31866595 & -2.95515086 & 3.04872188 \\
\hline $\mathrm{H}$ & 3.23350416 & -1.62463811 & -3.61012650 \\
\hline $\mathrm{H}$ & -1.38688962 & 4.94487827 & -0.02067468 \\
\hline $\mathrm{H}$ & -2.02054278 & -0.70513938 & -2.45041838 \\
\hline $\mathrm{H}$ & 1.34826077 & 3.96666327 & 2.89478010 \\
\hline $\mathrm{H}$ & -2.63964241 & 4.09316553 & -1.68911365 \\
\hline $\mathrm{H}$ & 2.82617441 & 1.58997260 & 3.94818832 \\
\hline $\mathrm{H}$ & -3.60144899 & 0.53971621 & -3.90100735 \\
\hline $\mathrm{H}$ & -3.91801854 & 3.00131408 & -3.51239045 \\
\hline
\end{tabular}


57

Geometry Os5

\begin{tabular}{|c|c|c|c|}
\hline Os & -0.09349845 & 0.00097663 & -0.00356413 \\
\hline $\mathrm{N}$ & -0.08700145 & 1.90849168 & 0.73423009 \\
\hline $\mathrm{N}$ & 1.29487231 & -0.12576946 & 1.56405327 \\
\hline $\mathrm{N}$ & -1.59320976 & -0.95002932 & 1.12627653 \\
\hline $\mathrm{N}$ & 1.42693057 & 0.11099935 & -1.44753671 \\
\hline $\mathrm{N}$ & -0.04445517 & -1.90698540 & -0.73901590 \\
\hline $\mathrm{N}$ & -1.47956807 & 0.96841439 & -1.25883811 \\
\hline $\mathrm{C}$ & -0.88507429 & -2.83118394 & -0.21216655 \\
\hline $\mathrm{C}$ & 0.06074304 & -4.49419254 & -1.66674759 \\
\hline $\mathrm{C}$ & -3.33715728 & -1.20056432 & 2.73246632 \\
\hline $\mathrm{C}$ & 1.66215938 & -1.08416755 & -2.09998594 \\
\hline $\mathrm{C}$ & -0.84856231 & -4.14842577 & -0.67013087 \\
\hline $\mathrm{C}$ & -1.76146078 & -2.28090786 & 0.83179234 \\
\hline $\mathrm{C}$ & 0.92339819 & -3.53367749 & -2.19293868 \\
\hline $\mathrm{C}$ & -2.38654479 & -0.42497215 & 2.07697438 \\
\hline $\mathrm{C}$ & -2.72982712 & -3.01719780 & 1.52017272 \\
\hline $\mathrm{C}$ & 0.85384042 & -2.23225740 & -1.70682315 \\
\hline $\mathrm{N}$ & -3.51112837 & -2.49380954 & 2.45882466 \\
\hline $\mathrm{C}$ & 2.66836621 & -0.83240066 & -3.00738199 \\
\hline $\mathrm{C}$ & 3.98159948 & 1.26951541 & -3.55275243 \\
\hline $\mathrm{C}$ & -0.86854436 & 2.84204631 & 0.13731439 \\
\hline $\mathrm{C}$ & 0.72844446 & 2.22353889 & 1.77604307 \\
\hline $\mathrm{C}$ & 1.48652527 & 1.06611115 & 2.23570945 \\
\hline $\mathrm{C}$ & -1.65742770 & 2.30124789 & -0.97910471 \\
\hline $\mathrm{C}$ & -0.85690928 & 4.15878367 & 0.59777528 \\
\hline $\mathrm{C}$ & -0.03302027 & 4.49419744 & 1.66952556 \\
\hline $\mathrm{C}$ & -2.19310321 & 0.45231130 & -2.27527241 \\
\hline $\mathrm{C}$ & 0.77045543 & 3.52436364 & 2.26723204 \\
\hline $\mathrm{C}$ & -2.55340815 & 3.04855444 & -1.74881604 \\
\hline $\mathrm{C}$ & 2.41023528 & 0.80191067 & 3.22465226 \\
\hline $\mathrm{C}$ & 3.63829193 & -1.32258589 & 3.87889379 \\
\hline $\mathrm{C}$ & -3.07377451 & 1.23871588 & -3.01075788 \\
\hline $\mathrm{N}$ & -3.25579562 & 2.53395630 & -2.75250106 \\
\hline $\mathrm{N}$ & 2.96942495 & 0.47733695 & -2.84596518 \\
\hline $\mathrm{N}$ & 2.21678249 & 1.04699039 & -1.89895527 \\
\hline $\mathrm{N}$ & 2.03329760 & -1.07149377 & 2.07908043 \\
\hline $\mathrm{N}$ & 2.70937176 & -0.51010114 & 3.08666086 \\
\hline $\mathrm{H}$ & 3.82485961 & 1.18689014 & -4.62738437 \\
\hline $\mathrm{H}$ & 3.86383869 & 2.30390698 & -3.24160758 \\
\hline $\mathrm{H}$ & 4.97794245 & 0.91464687 & -3.29049733 \\
\hline $\mathrm{H}$ & 4.43133970 & -1.69726409 & 3.23385881 \\
\hline $\mathrm{H}$ & 4.06309889 & -0.69760847 & 4.66089121 \\
\hline $\mathrm{H}$ & 3.09701706 & -2.15495844 & 4.32516833 \\
\hline $\mathrm{H}$ & 0.10016477 & -5.51384645 & -2.03076747 \\
\hline $\mathrm{H}$ & -3.97200444 & -0.76371737 & 3.49643388 \\
\hline $\mathrm{H}$ & -1.51180851 & -4.89833171 & -0.25890081 \\
\hline $\mathrm{H}$ & 1.63785578 & -3.79734413 & -2.96338078 \\
\hline $\mathrm{H}$ & -2.25195796 & 0.62403660 & 2.30648722 \\
\hline $\mathrm{H}$ & -2.87965135 & -4.06983559 & 1.30374042 \\
\hline $\mathrm{H}$ & 3.17357587 & -1.45543938 & -3.72754900 \\
\hline $\mathrm{H}$ & -1.47399867 & 4.91612290 & 0.13193776 \\
\hline $\mathrm{H}$ & -0.01389155 & 5.51333633 & 2.03661975 \\
\hline $\mathrm{H}$ & -2.05137360 & -0.59832174 & -2.49282236 \\
\hline $\mathrm{H}$ & 1.41868010 & 3.78027517 & 3.09661275 \\
\hline $\mathrm{H}$ & -2.70932497 & 4.10292695 & -1.54547091 \\
\hline $\mathrm{H}$ & 2.85891401 & 1.41958889 & 3.98530861 \\
\hline $\mathrm{H}$ & -3.64456524 & 0.80906601 & -3.82750160 \\
\hline
\end{tabular}


55

Geometry Os6

\begin{tabular}{|c|c|c|c|}
\hline Os & -0.09953590 & 0.00108472 & -0.00492679 \\
\hline $\mathrm{N}$ & -0.08596825 & 1.91354903 & 0.67984144 \\
\hline $\mathrm{N}$ & 1.28471810 & -0.08384062 & 1.57404955 \\
\hline $\mathrm{N}$ & -1.60762977 & -0.93606177 & 1.14314451 \\
\hline $\mathrm{N}$ & 1.42767195 & 0.06931471 & -1.44945043 \\
\hline $\mathrm{N}$ & -0.04226709 & -1.91204411 & -0.68559016 \\
\hline $\mathrm{N}$ & -1.48410663 & 0.95488368 & -1.28817258 \\
\hline $\mathrm{C}$ & -0.87501710 & -2.83240031 & -0.14482739 \\
\hline $\mathrm{N}$ & 0.07868790 & -4.52342082 & -1.53878717 \\
\hline $\mathrm{C}$ & -3.35896342 & -1.16631767 & 2.74364068 \\
\hline $\mathrm{C}$ & 1.67016353 & -1.14327059 & -2.06772571 \\
\hline $\mathrm{C}$ & -0.79025255 & -4.15310122 & -0.60004451 \\
\hline $\mathrm{C}$ & -1.76391659 & -2.27532847 & 0.87924243 \\
\hline $\mathrm{C}$ & 0.89482568 & -3.60266696 & -2.05218104 \\
\hline $\mathrm{C}$ & -2.41032876 & -0.39607336 & 2.07617380 \\
\hline $\mathrm{C}$ & -2.72946120 & -3.00654969 & 1.57705188 \\
\hline $\mathrm{C}$ & 0.85788422 & -2.27125440 & -1.63767382 \\
\hline $\mathrm{N}$ & -3.51981878 & -2.46638624 & 2.49838888 \\
\hline $\mathrm{C}$ & 2.68053569 & -0.91335120 & -2.97662589 \\
\hline $\mathrm{C}$ & 3.98878007 & 1.17811632 & -3.57449844 \\
\hline $\mathrm{C}$ & -0.85520919 & 2.84284947 & 0.06535165 \\
\hline $\mathrm{C}$ & 0.72481037 & 2.26218434 & 1.71273321 \\
\hline $\mathrm{C}$ & 1.48108301 & 1.12521896 & 2.21428091 \\
\hline $\mathrm{C}$ & -1.64983146 & 2.29569981 & -1.03845502 \\
\hline $\mathrm{C}$ & -0.80057776 & 4.16193935 & 0.52947840 \\
\hline $\mathrm{N}$ & -0.01954452 & 4.52217201 & 1.54628112 \\
\hline $\mathrm{C}$ & -2.20162950 & 0.42398899 & -2.29299446 \\
\hline $\mathrm{C}$ & 0.73593769 & 3.59280715 & 2.13172130 \\
\hline $\mathrm{C}$ & -2.53677480 & 3.03788566 & -1.82355614 \\
\hline $\mathrm{C}$ & 2.40197892 & 0.88308124 & 3.21182633 \\
\hline $\mathrm{C}$ & 3.61858188 & -1.22972921 & 3.92483669 \\
\hline $\mathrm{C}$ & -3.07439006 & 1.20489120 & -3.04613493 \\
\hline $\mathrm{N}$ & -3.24267320 & 2.50675277 & -2.81599223 \\
\hline $\mathrm{N}$ & 2.97577378 & 0.40130573 & -2.84909980 \\
\hline $\mathrm{N}$ & 2.21671275 & 0.99444137 & -1.92258390 \\
\hline $\mathrm{N}$ & 2.01744909 & -1.01834654 & 2.11546304 \\
\hline $\mathrm{N}$ & 2.69428974 & -0.43344230 & 3.10854489 \\
\hline $\mathrm{H}$ & 3.82662569 & 1.07645190 & -4.64663665 \\
\hline $\mathrm{H}$ & 3.87590195 & 2.21814197 & -3.28092729 \\
\hline $\mathrm{H}$ & 4.98409570 & 0.82304580 & -3.30924796 \\
\hline $\mathrm{H}$ & 4.40869449 & -1.62734467 & 3.29016959 \\
\hline $\mathrm{H}$ & 4.04695298 & -0.58484210 & 4.68841792 \\
\hline $\mathrm{H}$ & 3.07102413 & -2.04506036 & 4.39416306 \\
\hline $\mathrm{H}$ & -4.00208058 & -0.71764585 & 3.49370555 \\
\hline $\mathrm{H}$ & -1.43133740 & -4.93280849 & -0.20265998 \\
\hline $\mathrm{H}$ & 1.59507719 & -3.93861462 & -2.81071010 \\
\hline $\mathrm{H}$ & -2.28747197 & 0.65882888 & 2.28457696 \\
\hline $\mathrm{H}$ & -2.86788614 & -4.06536332 & 1.38289176 \\
\hline $\mathrm{H}$ & 3.19093258 & -1.55510369 & -3.67666485 \\
\hline $\mathrm{H}$ & -1.39431127 & 4.94855402 & 0.07612830 \\
\hline $\mathrm{H}$ & -2.07212248 & -0.63235281 & -2.48969616 \\
\hline $\mathrm{H}$ & 1.36539752 & 3.92090742 & 2.95313603 \\
\hline $\mathrm{H}$ & -2.68082064 & 4.09824424 & -1.64238081 \\
\hline $\mathrm{H}$ & 2.85201538 & 1.51959572 & 3.95620599 \\
\hline $\mathrm{H}$ & -3.64929776 & 0.76342994 & -3.85366153 \\
\hline
\end{tabular}


Geometry Os7

$\begin{array}{lll}0.00000000 & 0.00000000 & 4.76510725\end{array}$

$\begin{array}{lll}0.00000000 & 0.00000000 & -4.76510725\end{array}$

$\begin{array}{lll}0.81052690 & 0.81052690 & 4.08604274\end{array}$

$\begin{array}{lll}-0.81052690 & -0.81052690 & 4.08604274\end{array}$

$\begin{array}{lll}0.81052690 & -0.81052690 & -4.08604274\end{array}$

$\begin{array}{lll}-0.81052690 & 0.81052690 & -4.08604274\end{array}$

$\begin{array}{lll}0.83447019 & 0.83447019 & 2.68759544\end{array}$

$\begin{array}{lll}-0.83447019 & -0.83447019 & 2.68759544\end{array}$

$\begin{array}{lll}0.83447019 & -0.83447019 & -2.68759544\end{array}$

$\begin{array}{llll}-0.83447019 & 0.83447019 & -2.68759544\end{array}$

$\begin{array}{lll}0.00000000 & 0.00000000 & 2.01832301\end{array}$

$\begin{array}{llll}0.00000000 & 0.00000000 & -2.01832301\end{array}$

$\begin{array}{lll}1.65977589 & 1.65977589 & 1.80047796\end{array}$

$\begin{array}{lll}-1.65977589 & -1.65977589 & 1.80047796\end{array}$

$\begin{array}{lll}1.65977589 & -1.65977589 & -1.80047796\end{array}$

$\begin{array}{lll}-1.65977589 & 1.65977589 & -1.80047796\end{array}$

$\begin{array}{lll}2.60002691 & 2.60002691 & 2.23277537\end{array}$

$\begin{array}{lll}-2.60002691 & -2.60002691 & 2.23277537\end{array}$

$\begin{array}{lll}2.60002691 & -2.60002691 & -2.23277537\end{array}$

$\begin{array}{lll}-2.60002691 & 2.60002691 & -2.23277537\end{array}$

$\begin{array}{lll}3.32906863 & 3.32906863 & 1.39476271\end{array}$

$\begin{array}{lll}-3.32906863 & -3.32906863 & 1.39476271\end{array}$

$\begin{array}{lll}3.32906863 & -3.32906863 & -1.39476271\end{array}$

$\begin{array}{lll}-3.32906863 & 3.32906863 & -1.39476271\end{array}$

$\begin{array}{lll}3.13228495 & 3.13228495 & 0.09211099\end{array}$

$\begin{array}{lll}-3.13228495 & -3.13228495 & 0.09211099\end{array}$

$\begin{array}{lll}3.13228495 & -3.13228495 & -0.09211099\end{array}$

$\begin{array}{lll}-3.13228495 & 3.13228495 & -0.09211099\end{array}$

$\begin{array}{llll}2.20703109 & 2.20703109 & -0.38715699\end{array}$

$\begin{array}{lll}-2.20703109 & -2.20703109 & -0.38715699\end{array}$

$\begin{array}{lll}2.20703109 & -2.20703109 & 0.38715699\end{array}$

$\begin{array}{lll}-2.20703109 & 2.20703109 & 0.38715699\end{array}$

$\begin{array}{lll}1.46655541 & 1.46655541 & 0.45429647\end{array}$

$\begin{array}{lll}-1.46655541 & -1.46655541 & 0.45429647\end{array}$

$\begin{array}{lll}1.46655541 & -1.46655541 & -0.45429647\end{array}$

$\begin{array}{lll}-1.46655541 & 1.46655541 & -0.45429647\end{array}$

$\begin{array}{lll}1.45395072 & 1.45395072 & 4.67704621\end{array}$

$\begin{array}{lll}-1.45395072 & -1.45395072 & 4.67704621\end{array}$

$\begin{array}{lll}1.45395072 & -1.45395072 & -4.67704621\end{array}$

$\begin{array}{lll}-1.45395072 & 1.45395072 & -4.67704621\end{array}$

$\begin{array}{lll}2.76861272 & 2.76861272 & 3.29170019\end{array}$

$\begin{array}{lll}-2.76861272 & -2.76861272 & 3.29170019\end{array}$

$\begin{array}{lll}2.76861272 & -2.76861272 & -3.29170019\end{array}$

$\begin{array}{lll}-2.76861272 & 2.76861272 & -3.29170019\end{array}$

$\begin{array}{lll}3.72497330 & 3.72497330 & -0.59710180\end{array}$

$\begin{array}{lll}-3.72497330 & -3.72497330 & -0.59710180\end{array}$

$\begin{array}{lll}3.72497330 & -3.72497330 & 0.59710180\end{array}$

$\begin{array}{lll}-3.72497330 & 3.72497330 & 0.59710180\end{array}$

$\begin{array}{llll}2.05564519 & 2.05564519 & -1.44809652\end{array}$

$\begin{array}{lll}-2.05564519 & -2.05564519 & -1.44809652\end{array}$

$\begin{array}{lll}2.05564519 & -2.05564519 & 1.44809652\end{array}$

$\begin{array}{lrrr}\mathrm{H} & -2.05564519 & 2.05564519 & 1.44809652 \\ \mathrm{Os} & 0.00000000 & 0.00000000 & 0.00000000\end{array}$ 